Article

\title{
Solutions to Fredholm Integral Inclusions via Generalized Fuzzy Contractions
}

\author{
Hamed H Al-Sulami ${ }^{1}$, Jamshaid Ahmad ${ }^{2, *}$, Nawab Hussain ${ }^{1}$ ) and Abdul Latif ${ }^{1}$ \\ 1 Department of Mathematics, King Abdulaziz University, P.O. Box 80203, Jeddah 21589, Saudi Arabia \\ 2 Department of Mathematics, University of Jeddah, P.O. Box 80327, Jeddah 21589, Saudi Arabia \\ * Correspondence: jkhan@uj.edu.sa; Tel.: +966-569-765-680
}

Received: 28 June 2019; Accepted: 19 August 2019; Published: 2 September 2019

check for updates

\begin{abstract}
The aim of this study is to investigate the existence of solutions for the following Fredholm integral inclusion $\varphi(t) \in\left[f(t)+\int_{0}^{1} K(t, s, \varphi(s)) \varrho s\right]$ for $t \in[0,1]$, where $f \in C[0,1]$ is a given real-valued function and $K:[0,1] \times[0,1] \times \mathbb{R} \rightarrow K_{c v}(\mathbb{R})$ a given multivalued operator, where $K_{c v}$ represents the family of non-empty compact and convex subsets of $\mathbb{R}, \varphi \in C[0,1]$ is the unknown function and $\varrho$ is a metric defined on $C[0,1]$. To attain this target, we take advantage of fixed point theorems for $\alpha$-fuzzy mappings satisfying a new class of contractive conditions in the context of complete metric spaces. We derive new fixed point results which extend and improve the well-known results of Banach, Kannan, Chatterjea, Reich, Hardy-Rogers, Berinde and Ćirić by means of this new class of contractions. We also give a significantly non-trivial example to support our new results.
\end{abstract}

Keywords: fredholm integral inclusion; $\alpha$-fuzzy mappings; $\Theta$-contractions; fixed point; multivalued mappings

MSC: 46S40; 47H10; 54H25

\section{Introduction and Preliminaries}

In 1922, Stefan Banach [1] established a prominent fixed point result for contractive mappings in complete metric space $(Q, \varrho)$.

Definition 1. ([1]) A mapping $Q: \mathcal{R} \rightarrow \mathcal{R}$ is called a contraction if $\exists \lambda \in[0,1)$ such that

$$
\varrho(Q \varphi, Q \psi) \leq \lambda \varrho(\varphi, \psi)
$$

$\forall \varphi, \psi \in Q$.

Jleli and Samet [2] introduced a new type of contraction and established some new fixed point theorems for such contraction in the context of generalized metric spaces.

Definition 2. Let $\Theta:(0, \infty) \rightarrow(1, \infty)$ be a function satisfying:

$\left(\Theta_{1}\right) \quad \Theta$ is nondecreasing.

$\left(\Theta_{2}\right)$ For each sequence $\left\{\alpha_{n}\right\} \subseteq R^{+}, \lim _{n \rightarrow \infty} \Theta\left(\alpha_{n}\right)=1$ if and only if $\lim _{n \rightarrow \infty}\left(\alpha_{n}\right)=0$.

$\left(\Theta_{3}\right) \quad$ There exist $0<h<1$ and $l \in(0, \infty]$ such that $\lim _{a \rightarrow 0^{+}} \frac{\Theta(\alpha)-1}{\alpha^{h}}=l$. 
A mapping $Q: \mathcal{R} \rightarrow \mathcal{R}$ is said to be $\Theta$-contraction if there exist the function $\Theta$ satisfying $\left(\Theta_{1}\right)-\left(\Theta_{3}\right)$ and a constant $k \in(0,1)$ such that ,

$$
\varrho(Q \varphi, Q \psi) \neq 0 \Longrightarrow \Theta(\varrho(Q \varphi, Q \psi)) \leq[\Theta(\varrho(\varphi, \psi))]^{k}
$$

$\forall \varphi, \psi \in Q$. We denote by $\digamma$ the family of functions $\Theta$ satisfying $\left(\Theta_{1}\right)-\left(\Theta_{3}\right)$ and which are continuous from the right. For more details in this direction, we refer the following [3-8] to the reader.

In 1969, Nadler [9] initiated the notion of multi-valued contraction and extended Banach contraction principle from single-valued mapping to multivalued mapping.

Definition 3. ([9]) A point $\varphi \in \mathcal{R}$ is called a fixed point of the multi-valued mapping $Q: \mathcal{R} \rightarrow 2^{\mathcal{R}}$ if $\varphi \in Q \varphi$.

For $\Omega_{1}, \Omega_{2} \in C(\mathcal{R})$, let $H: C(\mathcal{R}) \times C(\mathcal{R}) \rightarrow[0, \infty)$ be defined by

$$
H\left(\Omega_{1}, \Omega_{2}\right)=\max \left\{\sup _{\varphi \in \Omega_{1}} \varrho\left(\varphi, \Omega_{2}\right), \sup _{\psi \in \Omega_{2}} \varrho\left(\psi, \Omega_{1}\right)\right\}
$$

where $\varrho\left(\varphi, \Omega_{2}\right)=\inf \left\{\vartheta(\varphi, \psi): \psi \in \Omega_{2}\right\}$. Such $H$ is called the generalized Hausdorff-Pompieu metric induced by the metric $\varrho$ and $2^{\mathcal{R}}, C L(\mathcal{R})$ and $C B(\mathcal{R})$ indicate the classes of all nonempty, closed and closed and bounded subsets of $\mathcal{R}$, respectively.

Definition 4. ([9]) A mapping $Q: \mathcal{R} \rightarrow C B(\mathcal{R})$ is said to be a multi-valued contraction if $\exists 0 \leq \lambda<1$ such that

$$
H(Q \varphi, Q \psi) \leq \lambda \varrho(\varphi, \psi)
$$

$\forall \varphi, \psi \in Q$.

Theorem 1. ([9]) Let $(Q, \varrho)$ be a complete metric space and $Q: \mathcal{R} \rightarrow C B(\mathcal{R})$ a multi-valued contraction; then, $Q$ has a fixed point.

In 1994, Constantin [10] introduced a new family $\mathcal{P}$ of continuous functions $\rho:\left(\mathbb{R}^{+}\right)^{5} \rightarrow \mathbb{R}^{+}$ satisfying the following assertions:

$\left(\rho_{1}\right) \quad \rho(1,1,1,2,0), \rho(1,1,1,0,2), \rho(1,1,1,1,1) \in(0,1]$.

$\left(\rho_{2}\right) \quad \rho$ is sub-homogeneous, that is, for all $\left(\varphi_{1}, \varphi_{2}, \varphi_{3}, \varphi_{4}, \varphi_{5}\right) \in\left(\mathbb{R}^{+}\right)^{5}$ and $\alpha \geq 0$, we have $\rho\left(\alpha \varphi_{1}, \alpha \varphi_{2}, \alpha \varphi_{3}, \alpha \varphi_{4}, \alpha \varphi_{5}\right) \leq \alpha \rho\left(\varphi_{1}, \varphi_{2}, \varphi_{3}, \varphi_{4}, \varphi_{5}\right)$.

$\left(\rho_{3}\right) \quad \rho$ is a non-decreasing function, that is, for $\varphi_{i}, \psi_{i} \in \mathbb{R}^{+}, \varphi_{i} \leq \psi_{i}, i=1, \ldots, 5$, we have

$$
\rho\left(\varphi_{1}, \varphi_{2}, \varphi_{3}, \varphi_{4}, \varphi_{5}\right) \leq \rho\left(\psi_{1}, \psi_{2}, \psi_{3}, \psi_{4}, \psi_{5}\right)
$$

and if $\varphi_{i}, \psi_{i} \in \mathbb{R}^{+}, i=1, \ldots, 4$, then $\rho\left(\varphi_{1}, \varphi_{2}, \varphi_{3}, \varphi_{4}, 0\right) \leq \rho\left(\psi_{1}, \psi_{2}, \psi_{3}, \psi_{4}, 0\right)$ and $\rho\left(\varphi_{1}, \varphi_{2}, \varphi_{3}, 0, \varphi_{4}\right) \leq \rho\left(\psi_{1}, \psi_{2}, \psi_{3}, 0, \psi_{4}\right)$.

Constantin also obtained a random fixed point theorem for multivalued mappings. Isik [11] utilized the above-mentioned family $\mathcal{P}$ of functions and established fixed point theorem for multivalued mappings in complete metric space.

On the other hand, Heilpern [12] employed the conception of fuzzy set to introduce a family of fuzzy mappings to generalize the multivalued mapping and obtained a fixed point result for fuzzy contractions in the setting of metric linear space. It is worth noting that the theorem given by Heilpern [12] is a real 
generalization of Nadler's result in the sense of fuzzy mappings. Moreover, we use the following notations that have been recorded from [13-20]:

A fuzzy set in $\mathcal{R}$ is a mapping with domain $\mathcal{R}$ and values in $[0,1]$. If $\Omega_{1}$ is a fuzzy set and $\varphi \in \mathcal{R}$, then $\Omega_{1}(\varphi)$ is called the grade of membership of $\varphi$ in $\Omega_{1}$. The $\alpha$-level set of $\Omega_{1}$ is denoted by $\left[\Omega_{1}\right]_{\alpha}$ and is defined in this way:

$$
\begin{gathered}
{\left[\Omega_{1}\right]_{\alpha}=\left\{\varphi: \Omega_{1}(\varphi) \geq \alpha\right\} \text { if } \alpha \in(0,1],} \\
{\left[\Omega_{1}\right]_{0}=\overline{\left\{\varphi: \Omega_{1}(\varphi)>0\right\} .}}
\end{gathered}
$$

Here, $\overline{\Omega_{2}}$ denotes the closure of the set $\Omega_{2}$. Let $(\mathcal{R})$ be the collection of all fuzzy sets in a metric space $\mathcal{R}$.

Definition 5. [15] Let $Q_{1}, Q_{2}: \mathcal{R} \rightarrow(\mathcal{R})$. A point $\varphi \in \mathcal{R}$ is called a common $\alpha$ - fuzzy fixed point of $Q_{1}$ and $Q_{2}$ if $\exists \alpha \in[0,1]$ so that $\varphi \in\left[Q_{1} \varphi\right]_{\alpha} \cap\left[Q_{2} \varphi\right]_{\alpha}$. If $\alpha=1$, then it reduces to common fixed point of $Q_{1}, Q_{2}: \mathcal{R} \rightarrow(\mathcal{R})$.

Remark 1. Let $(\mathcal{R}, \varrho)$ be a metric space and let $\mathcal{Q}_{1}, \mathcal{Q}_{2}: \mathcal{R} \rightarrow(\mathcal{R})$ and for each $\varphi, \psi \in \mathcal{R}$, $\exists \alpha_{\mathcal{Q}_{1}}(\varphi), \alpha_{\mathcal{Q}_{2}}(\psi) \in(0,1]$ such that $\left[\mathcal{Q}_{1} \varphi\right]_{\alpha_{\mathcal{Q}_{1}}(\varphi)},\left[\mathcal{Q}_{2} \psi\right]_{\alpha_{\mathcal{Q}_{2}}(\psi)} \in C B(\mathcal{R})$. Assume that there exist some $\Theta \in \digamma$ and $k \in(0,1)$ such that

$$
\Theta\left(H\left(\left[\mathcal{Q}_{1} \varphi\right]_{\alpha_{\mathcal{Q}_{1}}(\varphi)},\left[\mathcal{Q}_{2} \psi\right]_{\mathcal{Q}_{\mathcal{Q}_{2}}(\psi)}\right)\right) \leq\left[\Theta\left(\rho\left(\begin{array}{c}
\varrho(\varphi, \psi), \varrho\left(\varphi,\left[\mathcal{Q}_{1} \varphi\right]_{\alpha_{\mathcal{Q}_{1}}(\varphi)}\right), \varrho\left(\psi,\left[\mathcal{Q}_{2} \psi\right]_{\alpha_{\mathcal{Q}_{2}}(\psi)}\right), \\
\varrho\left(\varphi,\left[\mathcal{Q}_{2} \psi\right]_{\alpha_{\mathcal{Q}_{2}}(\psi)}\right), \varrho\left(\psi,\left[\mathcal{Q}_{1} \varphi\right]_{\alpha_{\mathcal{Q}_{1}}(\varphi)}\right)
\end{array}\right)\right]^{k}\right.
$$

for all $\varphi, \psi \in \mathcal{R}$ with $H\left(\left[\mathcal{Q}_{1} \varphi\right]_{\alpha_{\mathcal{Q}_{1}}(\varphi)},\left[\mathcal{Q}_{2} \psi\right]_{\alpha_{\mathcal{Q}_{2}}(\psi)}\right)>0$.

Then, from Inequation (4), we get

$$
\begin{aligned}
\Theta\left(H\left(\left[\mathcal{Q}_{1} \varphi\right]_{\alpha_{\mathcal{Q}_{1}}(\varphi),}\left[\mathcal{Q}_{2} \psi\right]_{\mathcal{Q}_{\mathcal{Q}_{2}}(\psi)}\right)\right) & \leq\left[\Theta\left(\rho\left(\begin{array}{c}
\varrho(\varphi, \psi), \varrho\left(\varphi,\left[\mathcal{Q}_{1} \varphi\right]_{\mathcal{Q}_{\mathcal{Q}_{1}}(\varphi)}\right), \varrho\left(\psi,\left[\mathcal{Q}_{2} \psi\right]_{\alpha_{\mathcal{Q}_{2}}(\psi)}\right), \\
\varrho\left(\varphi,\left[\mathcal{Q}_{2} \psi\right]_{\mathcal{Q}_{\mathcal{Q}_{2}}(\psi)}\right), \varrho\left(\psi,\left[\mathcal{Q}_{1} \varphi\right]_{\mathcal{Q}_{\mathcal{Q}_{1}(\varphi)}}\right)
\end{array}\right)\right)\right]^{k} \\
& <\Theta\left(\rho\left(\begin{array}{c}
\varrho(\varphi, \psi), \varrho\left(\varphi,\left[\mathcal{Q}_{1} \varphi\right]_{\mathcal{Q}_{\mathcal{Q}_{1}}(\varphi)}\right), \varrho\left(\psi,\left[\mathcal{Q}_{2} \psi\right]_{\alpha_{\mathcal{Q}_{2}}(\psi)}\right), \\
\varrho\left(\varphi,\left[\mathcal{Q}_{2} \psi\right]_{\mathcal{Q}_{\mathcal{Q}_{2}}(\psi)}\right), \varrho\left(\psi,\left[\mathcal{Q}_{1} \varphi\right]_{\left.\alpha_{\mathcal{Q}_{1}(\varphi)}\right)}\right)
\end{array}\right) .\right.
\end{aligned}
$$

Since $\Theta$ is non-decreasing, we obtain

$$
H\left(\left[\mathcal{Q}_{1} \varphi\right]_{\alpha_{\mathcal{Q}_{1}}(\varphi),},\left[\mathcal{Q}_{2} \psi\right]_{\alpha_{\mathcal{Q}_{2}}(\psi)}\right)<\rho\left(\begin{array}{c}
\varrho(\varphi, \psi), \varrho\left(\varphi,\left[\mathcal{Q}_{1} \varphi\right]_{\alpha_{\mathcal{Q}_{1}}(\varphi)}\right), \varrho\left(\psi,\left[\mathcal{Q}_{2} \psi\right]_{\alpha_{\mathcal{Q}_{2}}(\psi)}\right), \\
\varrho\left(\varphi,\left[\mathcal{Q}_{2} \psi\right]_{\mathcal{Q}_{\mathcal{Q}_{2}}(\psi)}\right), \varrho\left(\psi,\left[\mathcal{Q}_{1} \varphi\right]_{\alpha_{\mathcal{Q}_{1}}(\varphi)}\right)
\end{array}\right)
$$

for all $\varphi, \psi \in \mathcal{R}$ with $H\left(\left[\mathcal{Q}_{1} \varphi\right]_{\alpha_{\mathcal{Q}_{1}}(\varphi)},\left[\mathcal{Q}_{2} \psi\right]_{\alpha_{\mathcal{Q}_{2}}(\psi)}\right)$.

We need the following lemmas of [11] in the proof of our main result.

Lemma 1. [11] If $\rho \in \mathcal{P}$ and $\varphi, v \in \mathbb{R}^{+}$are such that

$$
\varphi<\max \{\rho(\psi, \psi, \varphi, \psi+\varphi, 0), \rho(\psi, \psi, \varphi, 0, \psi+\varphi), \rho(\psi, \varphi, \psi, \psi+\varphi, 0), \rho(\psi, \varphi, \psi, 0, \psi+\varphi)\},
$$

then $\varphi<\psi$. 
Lemma 2. [11] Let $(\mathcal{R}, \varrho)$ be a metric space and $\Omega_{1}, \Omega_{2} \in C L(\mathcal{R})$ with $H\left(\Omega_{1}, \Omega_{2}\right)>0$. Then, for each $h>1$ and for each $\varphi \in \Omega_{1}$, there exists $\psi=\psi(\varphi) \in \Omega_{2}$ such that $\varrho(\varphi, \psi)<h H\left(\Omega_{1}, \Omega_{2}\right)$.

In this paper, we obtain certain new fixed point theorems for $\alpha$-fuzzy mappings satisfying a new class of contractive conditions in the context of complete metric space. We also investigate the existence of solutions for Fredholm integral inclusion. We also provide a non-trivial example to support our new results.

\section{Main Results}

Theorem 2. Let $(\mathcal{R}, \varrho)$ be a metric space and let $\mathcal{Q}_{1}, \mathcal{Q}_{2}: \mathcal{R} \rightarrow(\mathcal{R})$ and for each $\varphi, \psi \in \mathcal{R}$, $\exists \alpha_{\mathcal{Q}_{1}}(\varphi), \alpha_{\mathcal{Q}_{2}}(\psi) \in(0,1]$ such that $\left[\mathcal{Q}_{1} \varphi\right]_{\alpha_{\mathcal{Q}_{1}}(\varphi)},\left[\mathcal{Q}_{2} \psi\right]_{\alpha_{\mathcal{Q}_{2}}(\psi)} \in C B(\mathcal{R})$. Assume that there exist some $\Theta \in \digamma$ and $k \in(0,1)$ such that

$$
\Theta\left(H\left(\left[\mathcal{Q}_{1} \varphi\right]_{\alpha_{\mathcal{Q}_{1}}(\varphi)},\left[\mathcal{Q}_{2} \psi\right]_{\mathcal{Q}_{\mathcal{Q}_{2}}(\psi)}\right)\right) \leq\left[\Theta\left(\rho\left(\begin{array}{c}
\varrho(\varphi, \psi), \varrho\left(\varphi,\left[\mathcal{Q}_{1} \varphi\right]_{\alpha_{\mathcal{Q}_{1}}(\varphi)}\right), \varrho\left(\psi,\left[\mathcal{Q}_{2} \psi\right]_{\alpha_{\mathcal{Q}_{2}}(\psi)}\right), \\
\varrho\left(\varphi,\left[\mathcal{Q}_{2} \psi\right]_{\alpha_{\mathcal{Q}_{2}}(\psi)}\right), \varrho\left(\psi,\left[\mathcal{Q}_{1} \varphi\right]_{\alpha_{\mathcal{Q}_{1}}(\varphi)}\right)
\end{array}\right)\right]^{k}\right.
$$

for all $\varphi, \psi \in \varphi$ with $H\left(\left[\mathcal{Q}_{1} \varphi\right]_{\mathcal{Q}_{1}(\varphi)},\left[\mathcal{Q}_{2} \psi\right]_{\alpha_{\mathcal{Q}_{2}}(\psi)}\right)>0$. Then, there exists $\varphi^{*} \in \mathcal{R}$ such that $\varphi^{*} \in\left[\mathcal{Q}_{1} \varphi^{*}\right]_{\alpha_{\mathcal{Q}_{1}}\left(\varphi^{*}\right)} \cap\left[\mathcal{Q}_{2} \varphi^{*}\right]_{\alpha_{\mathcal{Q}_{2}}\left(\varphi^{*}\right)} \cdot$

Proof. Let $\varphi_{0}$ be an arbitrary point in $\mathcal{R}$. By hypothesis there exists $\alpha_{\mathcal{Q}_{1}}\left(\varphi_{0}\right) \in(0,1]$ such that $\left[\mathcal{Q}_{1} \varphi_{0}\right]_{\alpha_{\mathcal{Q}_{1}}\left(\varphi_{0}\right)}$ is a nonempty, closed and bounded subset of $\mathcal{R}$. Let $\varphi_{1} \in\left[\mathcal{Q}_{1} \varphi_{0}\right]_{\alpha_{\mathcal{Q}_{1}}\left(\varphi_{0}\right)}$. For this $\varphi_{1}$, there exists $\alpha_{\mathcal{Q}_{2}}\left(\varphi_{1}\right) \in$ $(0,1]$ such that $\left[\mathcal{Q}_{2} \varphi_{1}\right]_{\mathcal{Q}_{\mathcal{Q}_{2}}\left(\varphi_{1}\right)}$ is a nonempty, closed and bounded subset of $\mathcal{R}$.

$$
\begin{aligned}
& \Theta\left(\varrho\left(\varphi_{1},\left[\mathcal{Q}_{2} \varphi_{1}\right]_{\alpha_{\mathcal{Q}_{2}}\left(\varphi_{1}\right)}\right)\right) \leq \Theta\left(H\left(\left[\mathcal{Q}_{1} \varphi_{0}\right]_{\alpha_{\mathcal{Q}_{1}}\left(\varphi_{0}\right)},\left[\mathcal{Q}_{2} \varphi_{1}\right]_{\alpha_{\mathcal{Q}_{2}}\left(\varphi_{1}\right)}\right)\right) \\
& \leq \Theta\left[\left(\rho\left(\begin{array}{c}
\varrho\left(\varphi_{0}, \varphi_{1}\right), \varrho\left(\varphi_{0},\left[\mathcal{Q}_{1} \varphi_{0}\right]_{\alpha_{\mathcal{Q}_{1}}\left(\varphi_{0}\right)}\right), \varrho\left(\varphi_{1},\left[\mathcal{Q}_{2} \varphi_{1}\right]_{\alpha_{\mathcal{Q}_{2}}\left(\varphi_{1}\right)}\right), \\
\varrho\left(\varphi_{0},\left[\mathcal{Q}_{2} \varphi_{1}\right]_{\alpha_{\mathcal{Q}_{2}}\left(\varphi_{1}\right)}\right), \varrho\left(\varphi_{1},\left[\mathcal{Q}_{1} \varphi_{0}\right]_{\mathcal{Q}_{\mathcal{Q}_{1}}\left(\varphi_{0}\right)}\right)
\end{array}\right)\right]^{k}\right. \\
& \leq\left[\Theta\left(\rho\left(\begin{array}{c}
\varrho\left(\varphi_{0}, \varphi_{1}\right), \varrho\left(\varphi_{0}, \varphi_{1}\right), \varrho\left(\varphi_{1},\left[\mathcal{Q}_{2} \varphi_{1}\right]_{\alpha_{\mathcal{Q}_{2}}\left(\varphi_{1}\right)}\right), \\
\varrho\left(\varphi_{0},\left[\mathcal{Q}_{2} \varphi_{1}\right]_{\alpha_{\mathcal{Q}_{2}}\left(\varphi_{1}\right)}\right), 0
\end{array}\right)\right]^{k}\right. \\
& <\Theta\left(\rho\left(\begin{array}{c}
\varrho\left(\varphi_{0}, \varphi_{1}\right), \varrho\left(\varphi_{0}, \varphi_{1}\right), \varrho\left(\varphi_{1},\left[\mathcal{Q}_{2} \varphi_{1}\right]_{\mathcal{Q}_{2}\left(\varphi_{1}\right)}\right), \\
\varrho\left(\varphi_{0},\left[\mathcal{Q}_{2} \varphi_{1}\right]_{\mathcal{Q}_{\mathcal{Q}_{2}}\left(\varphi_{1}\right)}\right), 0
\end{array}\right)\right)
\end{aligned}
$$

and so

$$
\varrho\left(\varphi_{1},\left[\mathcal{Q}_{2} \varphi_{1}\right]_{\alpha_{\mathcal{Q}_{2}}\left(\varphi_{1}\right)}\right)<\rho\left(\begin{array}{c}
\varrho\left(\varphi_{0}, \varphi_{1}\right), \varrho\left(\varphi_{0}, \varphi_{1}\right), \varrho\left(\varphi_{1},\left[\mathcal{Q}_{2} \varphi_{1}\right]_{\alpha_{\mathcal{Q}_{2}}\left(\varphi_{1}\right)}\right), \\
\varrho\left(\varphi_{0},\left[\mathcal{Q}_{2} \varphi_{1}\right]_{\alpha_{\mathcal{Q}_{2}}\left(\varphi_{1}\right)}\right), 0
\end{array}\right)
$$


Now, Lemma 1 gives that $\varrho\left(\varphi_{1},\left[\mathcal{Q}_{2} \varphi_{1}\right]_{\alpha_{\mathcal{Q}_{2}}\left(\varphi_{1}\right)}\right)<\varrho\left(\varphi_{0}, \varphi_{1}\right)$. Thus, we obtain

$$
\begin{aligned}
& \Theta\left(H \left(\left[\mathcal{Q}_{1} \varphi_{0}\right]_{\left.\left.\alpha_{\mathcal{Q}_{1}}\left(\varphi_{0}\right),\left[\mathcal{Q}_{2} \varphi_{1}\right]_{\alpha_{\mathcal{Q}_{2}}\left(\varphi_{1}\right)}\right)\right)}\right.\right. \\
\leq & {\left[\Theta\left(\rho\left(\begin{array}{c}
\left.\left.\varrho\left(\varphi_{0}, \varphi_{1}\right), \varrho\left(\varphi_{0},\left[\mathcal{Q}_{1} \varphi_{0}\right]_{\alpha_{\mathcal{Q}_{1}}\left(\varphi_{0}\right)}\right), \varrho\left(\varphi_{1},\left[\mathcal{Q}_{2} \varphi_{1}\right]_{\left.\alpha_{\mathcal{Q}_{2}\left(\varphi_{1}\right)}\right),}\right)\right)\right] \\
\varrho\left(\varphi_{0}, \varphi_{1}\right)+\varrho\left(\varphi_{1},\left[\mathcal{Q}_{2} \varphi_{1}\right]_{\mathcal{Q}_{\mathcal{Q}_{2}}\left(\varphi_{1}\right)}\right), 0
\end{array}\right)\right]^{k}\right.} \\
< & {\left[\Theta\left(\rho\left(\begin{array}{c}
\varrho\left(\varphi_{0}, \varphi_{1}\right), \varrho\left(\varphi_{0}, \varphi_{1}\right), \varrho\left(\varphi_{0}, \varphi_{1}\right), \\
2 \varrho\left(\varphi_{0}, \varphi_{1}\right), 0
\end{array}\right)\right]^{k}\right.} \\
\leq & {\left[\Theta\left(\varrho\left(\varphi_{0}, \varphi_{1}\right) \rho(1,1,1,2,0)\right)\right]^{k} } \\
\leq & {\left[\Theta\left(\varrho\left(\varphi_{0}, \varphi_{1}\right)\right)\right]^{k} }
\end{aligned}
$$

and hence

$$
\Theta\left(H\left(\left[\mathcal{Q}_{1} \varphi_{0}\right]_{\alpha_{\mathcal{Q}_{1}}\left(\varphi_{0}\right)},\left[\mathcal{Q}_{2} \varphi_{1}\right]_{\alpha_{\mathcal{Q}_{2}}\left(\varphi_{1}\right)}\right)\right)<\left[\Theta\left(\varrho\left(\varphi_{0}, \varphi_{1}\right)\right)\right]^{k} .
$$

Since $\Theta \in \digamma$ is continuous function from the right, there exists a real number $h>1$ such that

$$
\Theta\left(h H\left(\left[\mathcal{Q}_{1} \varphi_{0}\right]_{\alpha_{\mathcal{Q}_{1}}\left(\varphi_{0}\right)},\left[\mathcal{Q}_{2} \varphi_{1}\right]_{\alpha_{\mathcal{Q}_{2}}\left(\varphi_{1}\right)}\right)\right) \leq\left[\Theta\left(\varrho\left(\varphi_{0}, \varphi_{1}\right)\right)\right]^{k} .
$$

As

$$
\varrho\left(\varphi_{1},\left[\mathcal{Q}_{2} \varphi_{1}\right]_{\alpha_{\mathcal{Q}_{2}}\left(\varphi_{1}\right)}\right) \leq H\left(\left[\mathcal{Q}_{1} \varphi_{0}\right]_{\alpha_{\mathcal{Q}_{1}}\left(\varphi_{0}\right)},\left[\mathcal{Q}_{2} \varphi_{1}\right]_{\alpha_{\mathcal{Q}_{2}}\left(\varphi_{1}\right)}\right)<h H\left(\left[\mathcal{Q}_{1} \varphi_{0}\right]_{\alpha_{\mathcal{Q}_{1}}\left(\varphi_{0}\right)},\left[\mathcal{Q}_{2} \varphi_{1}\right]_{\alpha_{\mathcal{Q}_{2}}\left(\varphi_{1}\right)}\right),
$$

by Lemma 2, there exists $\varphi_{2} \in\left[\mathcal{Q}_{2} \varphi_{1}\right]_{\alpha_{\mathcal{Q}_{2}}\left(\varphi_{1}\right)}$ (obviously, $\left.\varphi_{2} \neq \varphi_{1}\right)$ such that

$$
\varrho\left(\varphi_{1}, \varphi_{2}\right) \leq h H\left(\left[\mathcal{Q}_{1} \varphi_{0}\right]_{\alpha_{\mathcal{Q}_{1}}\left(\varphi_{0}\right)},\left[\mathcal{Q}_{2} \varphi_{1}\right]_{\alpha_{\mathcal{Q}_{2}}\left(\varphi_{1}\right)}\right) .
$$

Thus by Inequalities (7) and (8), we have

$$
\Theta\left(\varrho\left(\varphi_{1}, \varphi_{2}\right)\right) \leq \Theta\left(h H\left(\left[\mathcal{Q}_{1} \varphi_{0}\right]_{\alpha_{\mathcal{Q}_{1}}\left(\varphi_{0}\right)},\left[\mathcal{Q}_{2} \varphi_{1}\right]_{\alpha_{\mathcal{Q}_{2}}\left(\varphi_{1}\right)}\right)\right) \leq\left[\Theta\left(\varrho\left(\varphi_{0}, \varphi_{1}\right)\right)\right]^{k} .
$$

Thus, we have

$$
\Theta\left(\varrho\left(\varphi_{1}, \varphi_{2}\right)\right) \leq\left[\Theta\left(\varrho\left(\varphi_{0}, \varphi_{1}\right)\right)\right]^{k}
$$


Mathematics 2019, 7, 808

6 of 19

For $\varphi_{2}, \exists \alpha_{\mathcal{Q}_{1}}\left(\varphi_{2}\right) \in(0,1]$ such that $\left[\mathcal{Q}_{1} \varphi_{2}\right]_{\alpha_{\mathcal{Q}_{1}}\left(\varphi_{2}\right)} \in C B(\mathcal{R})$. Thus, we have

$$
\begin{aligned}
& \Theta\left(\varrho\left(\varphi_{2},\left[\mathcal{Q}_{1} \varphi_{2}\right]_{\alpha_{\mathcal{Q}_{1}}\left(\varphi_{2}\right)}\right)\right) \leq \Theta\left(H\left(\left[\mathcal{Q}_{2} \varphi_{1}\right]_{\alpha_{\mathcal{Q}_{2}}\left(\varphi_{1}\right)},\left[\mathcal{Q}_{1} \varphi_{2}\right]_{\alpha_{\mathcal{Q}_{1}}\left(\varphi_{2}\right)}\right)\right) \\
& =\Theta\left(H\left(\left[\mathcal{Q}_{1} \varphi_{2}\right]_{\mathcal{Q}_{\mathcal{Q}_{1}}\left(\varphi_{2}\right)},\left[\mathcal{Q}_{2} \varphi_{1}\right]_{\mathcal{Q}_{\mathcal{Q}_{2}}\left(\varphi_{1}\right)}\right)\right) \\
& \leq\left[\Theta\left(\rho\left(\begin{array}{c}
\varrho\left(\varphi_{2}, \varphi_{1}\right), \varrho\left(\varphi_{2},\left[\mathcal{Q}_{1} \varphi_{2}\right]_{\alpha_{\mathcal{Q}_{1}}\left(\varphi_{2}\right)}\right), \varrho\left(\varphi_{1},\left[\mathcal{Q}_{2} \varphi_{1}\right]_{\alpha_{\mathcal{Q}_{2}}\left(\varphi_{1}\right)}\right), \\
\varrho\left(\varphi_{2},\left[\mathcal{Q}_{2} \varphi_{1}\right]_{\alpha_{\mathcal{Q}_{2}}\left(\varphi_{1}\right)}\right), \varrho\left(\varphi_{1},\left[\mathcal{Q}_{1} \varphi_{2}\right]_{\mathcal{Q}_{1}\left(\varphi_{2}\right)}\right)
\end{array}\right)\right]^{k}\right. \\
& \leq\left[\Theta\left(\rho\left(\begin{array}{c}
\varrho\left(\varphi_{2}, \varphi_{1}\right), \varrho\left(\varphi_{2},\left[\mathcal{Q}_{1} \varphi_{2}\right]_{\alpha_{\mathcal{Q}_{1}}\left(\varphi_{2}\right)}, \varrho\left(\varphi_{1}, \varphi_{2}\right),\right. \\
0, \varrho\left(\varphi_{1},\left[\mathcal{Q}_{1} \varphi_{2}\right]_{\alpha_{\mathcal{Q}_{1}}\left(\varphi_{2}\right)}\right.
\end{array}\right)\right)\right]^{k} \\
& =\left[\Theta\left(\rho\left(\begin{array}{c}
\varrho\left(\varphi_{1}, \varphi_{2}\right), \varrho\left(\varphi_{2},\left[\mathcal{Q}_{1} \varphi_{2}\right]_{\alpha_{\mathcal{Q}_{1}}\left(\varphi_{2}\right)}, \varrho\left(\varphi_{1}, \varphi_{2}\right),\right. \\
0, \varrho\left(\varphi_{1},\left[\mathcal{Q}_{1} \varphi_{2}\right]_{\mathcal{Q}_{\mathcal{Q}_{1}}\left(\varphi_{2}\right)}\right.
\end{array}\right)\right)\right]^{k} \\
& <\Theta\left(\rho\left(\begin{array}{c}
\varrho\left(\varphi_{1}, \varphi_{2}\right), \varrho\left(\varphi_{2},\left[\mathcal{Q}_{1} \varphi_{2}\right]_{\mathcal{Q}_{1}\left(\varphi_{2}\right)}, \varrho\left(\varphi_{1}, \varphi_{2}\right),\right. \\
0, \varrho\left(\varphi_{1},\left[\mathcal{Q}_{1} \varphi_{2}\right]_{\mathcal{Q}_{\mathcal{Q}_{1}}\left(\varphi_{2}\right)}\right.
\end{array}\right)\right)
\end{aligned}
$$

and so

$$
\varrho\left(\varphi_{2},\left[\mathcal{Q}_{1} \varphi_{2}\right]_{\mathcal{Q}_{1}\left(\varphi_{2}\right)}\right)<\rho\left(\begin{array}{c}
\varrho\left(\varphi_{1}, \varphi_{2}\right), \varrho\left(\varphi_{2},\left[\mathcal{Q}_{1} \varphi_{2}\right]_{\alpha_{\mathcal{Q}_{1}}\left(\varphi_{2}\right)}, \varrho\left(\varphi_{1}, \varphi_{2}\right),\right. \\
0, \varrho\left(\varphi_{1},\left[\mathcal{Q}_{1} \varphi_{2}\right]_{\mathcal{Q}_{\mathcal{Q}_{1}}\left(\varphi_{2}\right)}\right.
\end{array}\right) .
$$

Now, Lemma 1 gives that $\varrho\left(\varphi_{2},\left[\mathcal{Q}_{1} \varphi_{2}\right]_{\mathcal{Q}_{\mathcal{Q}_{1}}\left(\varphi_{2}\right)}\right)<\varrho\left(\varphi_{1}, \varphi_{2}\right)$. Thus, we obtain

$$
\begin{aligned}
& \Theta\left(H\left(\left[\mathcal{Q}_{2} \varphi_{1}\right]_{\mathcal{Q}_{2}\left(\varphi_{1}\right)},\left[\mathcal{Q}_{1} \varphi_{2}\right]_{\alpha_{\mathcal{Q}_{1}}\left(\varphi_{2}\right)}\right)\right)=\Theta\left(H\left(\left[\mathcal{Q}_{1} \varphi_{2}\right]_{\alpha_{\mathcal{Q}_{1}}\left(\varphi_{2}\right)},\left[\mathcal{Q}_{2} \varphi_{1}\right]_{\alpha_{\mathcal{Q}_{2}}\left(\varphi_{1}\right)}\right)\right) \\
& \leq\left[\Theta\left(\rho\left(\begin{array}{c}
\varrho\left(\varphi_{1}, \varphi_{2}\right), \varrho\left(\varphi_{2},\left[\mathcal{Q}_{1} \varphi_{2}\right]_{\alpha_{\mathcal{Q}_{1}}\left(\varphi_{2}\right)}, \varrho\left(\varphi_{1}, \varphi_{2}\right),\right. \\
0, \varrho\left(\varphi_{1},\left[\mathcal{Q}_{1} \varphi_{2}\right]_{\alpha_{\mathcal{Q}_{1}}\left(\varphi_{2}\right)}\right.
\end{array}\right)\right)\right]^{k} \\
& \leq\left[\Theta\left(\rho\left(\begin{array}{c}
\varrho\left(\varphi_{1}, \varphi_{2}\right), \varrho\left(\varphi_{1}, \varphi_{2}\right), \varrho\left(\varphi_{1}, \varphi_{2}\right), \\
0,2 \varrho\left(\varphi_{1}, \varphi_{2}\right)
\end{array}\right)\right)\right]^{k} \\
& \leq\left[\Theta\left(\varrho\left(\varphi_{1}, \varphi_{2}\right) \rho(1,1,1,0,2)\right)\right]^{k} \\
& \leq\left[\Theta\left(\varrho\left(\varphi_{1}, \varphi_{2}\right)\right)\right]^{k}
\end{aligned}
$$

and hence

$$
\Theta\left(H\left(\left[\mathcal{Q}_{2} \varphi_{1}\right]_{\alpha_{\mathcal{Q}_{2}}\left(\varphi_{1}\right)},\left[\mathcal{Q}_{1} \varphi_{2}\right]_{\alpha_{\mathcal{Q}_{1}}\left(\varphi_{2}\right)}\right)\right)<\left[\Theta\left(\varrho\left(\varphi_{1}, \varphi_{2}\right)\right)\right]^{k} .
$$

Since $\Theta \in \digamma$ is continuous function from the right, there exists a real number $h>1$ such that

$$
\Theta\left(h H\left(\left[\mathcal{Q}_{2} \varphi_{1}\right]_{\mathcal{Q}_{\mathcal{Q}_{2}}\left(\varphi_{1}\right)},\left[\mathcal{Q}_{1} \varphi_{2}\right]_{\mathcal{Q}_{\mathcal{Q}_{1}}\left(\varphi_{2}\right)}\right)\right) \leq\left[\Theta\left(\varrho\left(\varphi_{1}, \varphi_{2}\right)\right)\right]^{k} .
$$

As

$$
\varrho\left(\varphi_{2},\left[\mathcal{Q}_{1} \varphi_{2}\right]_{\alpha_{\mathcal{Q}_{1}}\left(\varphi_{2}\right)}\right) \leq H\left(\left[\mathcal{Q}_{2} \varphi_{1}\right]_{\alpha_{\mathcal{Q}_{2}}\left(\varphi_{1}\right)},\left[\mathcal{Q}_{1} \varphi_{2}\right]_{\alpha_{\mathcal{Q}_{1}}\left(\varphi_{2}\right)}\right)<h H\left(\left[\mathcal{Q}_{2} \varphi_{1}\right]_{\alpha_{\mathcal{Q}_{2}}\left(\varphi_{1}\right)},\left[\mathcal{Q}_{1} \varphi_{2}\right]_{\alpha_{\mathcal{Q}_{1}}\left(\varphi_{2}\right)}\right),
$$


by Lemma 2, there exists $\varphi_{3} \in\left[\mathcal{Q}_{1} \varphi_{2}\right]_{\alpha_{\mathcal{Q}_{1}}\left(\varphi_{2}\right)}$ (obviously, $\left.\varphi_{3} \neq \varphi_{2}\right)$ such that

$$
\varrho\left(\varphi_{2}, \varphi_{3}\right) \leq h H\left(\left[\mathcal{Q}_{2} \varphi_{1}\right]_{\alpha_{\mathcal{Q}_{2}}\left(\varphi_{1}\right)},\left[\mathcal{Q}_{1} \varphi_{2}\right]_{\alpha_{\mathcal{Q}_{1}}\left(\varphi_{2}\right)}\right) .
$$

Thus by Inequalities (11) and (12), we have

$$
\Theta\left(\varrho\left(\varphi_{2}, \varphi_{3}\right)\right) \leq \Theta\left(h H\left(\left[\mathcal{Q}_{2} \varphi_{1}\right]_{\alpha_{\mathcal{Q}_{2}}\left(\varphi_{1}\right)},\left[\mathcal{Q}_{1} \varphi_{2}\right]_{\mathcal{Q}_{1}\left(\varphi_{2}\right)}\right)\right) \leq\left[\Theta\left(\varrho\left(\varphi_{1}, \varphi_{2}\right)\right)\right]^{k} .
$$

Thus, we have

$$
\Theta\left(\varrho\left(\varphi_{2}, \varphi_{3}\right)\right) \leq\left[\Theta\left(\varrho\left(\varphi_{1}, \varphi_{2}\right)\right)\right]^{k} .
$$

Thus, pursuing repeatedly, we get $\left\{\varphi_{n}\right\}$ in $\mathcal{R}$ so that $\varphi_{2 n+1} \in\left[\mathcal{Q}_{1} \varphi_{2 n}\right]_{\alpha_{\mathcal{Q}_{1}}\left(\varphi_{2 n}\right)}, \varphi_{2 n+2} \in$ $\left[\mathcal{Q}_{2} \varphi_{2 n+1}\right]_{\alpha_{\mathcal{Q}_{2}}\left(\varphi_{2 n+1}\right)^{\prime}}$

$$
\Theta\left(\varrho\left(\varphi_{2 n+1}, \varphi_{2 n+2}\right)\right) \leq\left[\Theta\left(\varrho\left(\varphi_{2 n}, \varphi_{2 n+1}\right)\right)\right]^{k}
$$

and

$$
\Theta\left(\varrho\left(\varphi_{2 n+2}, \varphi_{2 n+3}\right)\right) \leq\left[\Theta\left(\varrho\left(\varphi_{2 n+1}, \varphi_{2 n+2}\right)\right)\right]
$$

$\forall n \in \mathbb{N}$. By Inequalities (14) and (15), we get

$$
1<\Theta\left(\varrho\left(\varphi_{n}, \varphi_{n+1}\right)\right) \leq\left[\Theta\left(\varrho\left(\varphi_{n-1}, \varphi_{n}\right)\right)\right]^{k}
$$

$\forall n \in \mathbb{N}$. Let $\sigma_{n}:=\varrho\left(\varphi_{n}, \varphi_{n+1}\right)$ for all $n \in \mathbb{N} \cup\{0\}$. From Inequality (16), we get

$$
1<\Theta\left(\sigma_{n}\right) \leq\left[\Theta\left(\sigma_{0}\right)\right]^{k^{n}}
$$

which implies that $\lim _{n \rightarrow \infty} \Theta\left(\sigma_{n}\right)=1$. By $\left(\Theta_{2}\right)$, we have $\lim _{n \rightarrow \infty} \sigma_{n}=0$. Now, we claim that $\left\{\varphi_{n}\right\}$ is a Cauchy sequence: for this, consider Condition $\left(\Theta_{3}\right)$. From $\left(\Theta_{3}\right)$, there exist $h \in(0,1)$ and $l \in(0, \infty)$ such that

$$
\lim _{n \rightarrow \infty} \frac{\Theta\left(\sigma_{n}\right)-1}{\left[\sigma_{n}\right]^{h}}=l
$$

Take $\lambda \in(0, l)$. By the definition of limit, there exists $n_{0} \in \mathbb{N}$ such that

$$
\left[\sigma_{n}\right]^{h} \leq \lambda^{-1}\left[\Theta\left(\sigma_{n}\right)-1\right]
$$

for all $n>n_{0}$. Using Inequality (17) and the above inequality, we deduce

$$
n\left[\sigma_{n}\right]^{h} \leq \lambda^{-1} n\left(\left[\Theta\left(\sigma_{0}\right)\right]^{k^{n}}-1\right) .
$$

This implies that

$$
\lim _{n \rightarrow \infty} n\left[\sigma_{n}\right]^{h}=\lim _{n \rightarrow \infty} n\left[\varrho\left(\varphi_{n}, \varphi_{n+1}\right)\right]^{h}=0 .
$$

Then, there exists $n_{1} \in \mathbb{N}$ such that

$$
\varrho\left(\varphi_{n}, \varphi_{n+1}\right) \leq \frac{1}{n^{\frac{1}{h}}}
$$


for $n>n_{1}$. Now, we prove that $\left\{\varphi_{n}\right\}$ is a Cauchy sequence. For $m>n>n_{1}$, we have

$$
\varrho\left(\varphi_{n}, \varphi_{m}\right) \leq \sum_{i=n}^{m-1} \varrho\left(\varphi_{i}, \varphi_{i+1}\right) \leq \sum_{i=n}^{m-1} \frac{1}{i^{1 / h}} \leq \sum_{i=1}^{\infty} \frac{1}{i^{1 / h}}
$$

Since, $0<h<1, \sum_{i=1}^{\infty} \frac{1}{i \frac{1}{h}}$ converges. Therefore, $\varrho\left(\varphi_{n}, \varphi_{m}\right) \rightarrow 0$ as $m, n \rightarrow \infty$. Thus, we proved that $\left\{\varphi_{n}\right\}$ is a Cauchy sequence in $\mathcal{R}$. The completeness of $\mathcal{R}$ ensures that there exists $\varphi^{*} \in \mathcal{R}$ such that, $\lim _{n \rightarrow \infty} \varphi_{n} \rightarrow \varphi^{*}$, that is

$$
\lim _{n \rightarrow \infty} \varphi_{n}=\varphi^{*} .
$$

Now, we prove that $\varphi^{*} \in\left[\mathcal{Q}_{2} \varphi^{*}\right]_{\alpha_{\mathcal{Q}_{2}}\left(\varphi^{*}\right)}$. Assuming on the contrary that $\varphi^{*} \notin\left[\mathcal{Q}_{2} \varphi^{*}\right]_{\alpha_{\mathcal{Q}_{2}}\left(\varphi^{*}\right)}$, then $\exists n_{2} \in \mathbb{N}$ and a subsequence $\left\{\varphi_{n_{k}}\right\}$ of $\left\{\varphi_{n}\right\}$ so that

$$
\varrho\left(\varphi_{2 n_{k}+1},\left[\mathcal{Q}_{2} \varphi^{*}\right]_{\alpha_{\mathcal{Q}_{2}}\left(\varphi^{*}\right)}\right)>0
$$

for all $n_{k} \geq n_{2}$. Now, using Inequality (5) with $\varphi=\varphi_{2 n_{k}+1}$ and $\psi=\varphi^{*}$. Taking Remark 1 into account, we have

$$
\begin{aligned}
\varrho\left(\varphi_{2 n_{k}+1},\left[\mathcal{Q}_{2} \varphi^{*}\right]_{\mathcal{Q}_{\mathcal{Q}_{2}}\left(\varphi^{*}\right)}\right) & \leq H\left(\left[\mathcal{Q}_{1} \varphi_{2 n_{k}}\right]_{\alpha_{\mathcal{Q}_{1}}\left(\varphi_{2 n_{k}}\right)},\left[\mathcal{Q}_{2} \varphi^{*}\right]_{\alpha_{\mathcal{Q}_{2}}\left(\varphi^{*}\right)}\right) \\
& \leq \rho\left(\begin{array}{c}
\varrho\left(\varphi_{2 n_{k}}, \varphi^{*}\right), \varrho\left(\varphi_{2 n_{k}},\left[\mathcal{Q}_{1} \varphi_{2 n_{k}}\right]_{\alpha_{\mathcal{Q}_{1}}\left(\varphi_{2 n_{k}}\right)}\right), \varrho\left(\varphi^{*},\left[\mathcal{Q}_{2} \varphi^{*}\right]_{\alpha_{\mathcal{Q}_{2}}\left(\varphi^{*}\right)}\right), \\
\varrho\left(\varphi_{2 n_{k}},\left[\mathcal{Q}_{2} \varphi^{*}\right]_{\alpha_{\mathcal{Q}_{2}}\left(\varphi^{*}\right)}\right), \varrho\left(\varphi^{*},\left[\mathcal{Q}_{1} \varphi_{2 n_{k}}\right]_{\left.\alpha_{\mathcal{Q}_{1}\left(\varphi_{2 n_{k}}\right)}\right)}\right) \\
\leq
\end{array}\right) \\
& \leq\left(\begin{array}{c}
\varrho\left(\varphi_{2 n_{k}}, \varphi^{*}\right), \varrho\left(\varphi_{2 n_{k}}, \varphi_{2 n_{k}+1}\right), \varrho\left(\varphi^{*},\left[\mathcal{Q}_{2} \varphi^{*}\right]_{\alpha_{\mathcal{Q}}\left(\varphi^{*}\right)}\right), \\
\varrho\left(\varphi_{2 n_{k}},\left[\mathcal{Q}_{2} \varphi^{*}\right]_{\alpha_{\mathcal{Q}_{2}}\left(\varphi^{*}\right)}\right), \varrho\left(\varphi^{*}, \varphi_{2 n_{k}+1}\right)
\end{array}\right) .
\end{aligned}
$$

Passing the limit as $n \rightarrow \infty$ in the above inequality, we obtain

$$
\varrho\left(\varphi^{*},\left[\mathcal{Q}_{2} \varphi^{*}\right]_{\alpha_{\mathcal{Q}_{2}}\left(\varphi^{*}\right)}\right) \leq \rho\left(0,0, \varrho\left(\varphi^{*},\left[\mathcal{Q}_{2} \varphi^{*}\right]_{\alpha_{\mathcal{Q}_{2}}\left(\varphi^{*}\right)}\right), \varrho\left(\varphi^{*},\left[\mathcal{Q}_{2} \varphi^{*}\right]_{\mathcal{Q}_{2}\left(\varphi^{*}\right)}\right), 0\right)
$$

which implies by Lemma 1 that

$$
0<\varrho\left(\varphi^{*},\left[\mathcal{Q}_{2} \varphi^{*}\right]_{\alpha_{\mathcal{Q}_{2}}\left(\varphi^{*}\right)}\right)<0
$$

which is a contradiction. Hence, $\varrho\left(\varphi^{*},\left[\mathcal{Q}_{2} \varphi^{*}\right]_{\alpha_{\mathcal{Q}_{2}}\left(\varphi^{*}\right)}\right)=0$. Since $\left[\mathcal{Q}_{2} \varphi^{*}\right]_{\mathcal{Q}_{2}\left(\varphi^{*}\right)}$ is closed, we deduce that

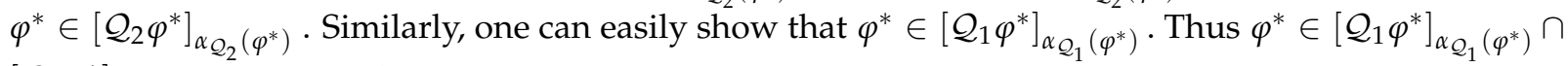
$\left[\mathcal{Q}_{2} \varphi^{*}\right]_{\alpha_{\mathcal{Q}_{2}}\left(\varphi^{*}\right)}$ as required.

Example 1. Let $\mathcal{R}=[0,1]$ and define $\varrho: \mathcal{R} \times \mathcal{R} \rightarrow \mathbb{R}^{+}$by

$$
\varrho(\varphi, \psi)=|\varphi-\psi|
$$

Clearly, $(\mathcal{R}, \varrho)$ satisfies all axioms of complete metric space. Define $\mathcal{Q}_{1}, \mathcal{Q}_{2}: \mathcal{R} \rightarrow(\mathcal{R})$ by

$$
\mathcal{Q}_{1}(\varphi)(t)=\left\{\begin{array}{l}
\alpha \text { if } 0 \leq t \leq \frac{\varphi}{16} \\
\frac{\alpha}{2} \text { if } \frac{\varphi}{16}<t \leq \frac{\varphi}{9} \\
\frac{\alpha}{3} \text { if } \frac{\varphi}{9}<t \leq \frac{\varphi}{4} \\
\frac{\alpha}{5} \text { if } \frac{\varphi}{4}<t \leq 1
\end{array}\right.
$$


and

$$
\mathcal{Q}_{2}(\varphi)(t)=\left\{\begin{array}{l}
\alpha \text { if } 0 \leq t \leq \frac{\varphi}{8} \\
\frac{\alpha}{3} \text { if } \frac{\varphi}{8}<t \leq \frac{\varphi}{5} \\
\frac{\alpha}{4} \text { if } \frac{\varphi}{5}<t \leq \frac{\varphi}{2} \\
\frac{\alpha}{7} \text { if } \frac{\varphi}{2}<t \leq 1
\end{array}\right.
$$

such that

$$
\begin{aligned}
{\left[\mathcal{Q}_{2} \varphi\right]_{\alpha} } & =\left[0, \frac{\varphi}{8}\right] \\
{\left[\mathcal{Q}_{1} \varphi\right]_{\alpha} } & =\left[0, \frac{\varphi}{16}\right]
\end{aligned}
$$

for $\alpha \in[0,1]$ and $\varphi \in \mathcal{R}$. Let $\rho:\left(\mathbb{R}^{+}\right)^{5} \rightarrow \mathbb{R}^{+}$be defined by $\rho\left(\varphi_{1}, \varphi_{2}, \varphi_{3}, \varphi_{4}, \varphi_{5}\right)=\max \left\{\varphi_{1}, \varphi_{2}, \varphi_{3}, \varphi_{4}, \varphi_{5}\right\}$ and $\Theta(t)=e^{\sqrt{t}}$. All the hypotheses of our main Theorem 2 are satisfied to obtain $0 \in\left[\mathcal{Q}_{1} 0\right]_{\alpha} \cap\left[\mathcal{Q}_{2} 0\right]_{\alpha}$ for $k=e^{-1} \in(0,1)$.

The following result for one fuzzy mapping is a direct consequence of our main theorem.

Corollary 1. Let $(\mathcal{R}, \varrho)$ be a complete metric space and let $\mathcal{Q}: \mathcal{R} \rightarrow(\mathcal{R})$ and for each $\varphi, \psi \in \mathcal{R}$, $\exists \alpha_{\mathcal{Q}}(\varphi), \alpha_{\mathcal{Q}}(\psi) \in(0,1]$ such that $[\mathcal{Q} \varphi]_{\alpha_{\mathcal{Q}}(\varphi)},[\mathcal{Q} \psi]_{\alpha_{\mathcal{Q}}(\psi)} \in C B(\mathcal{R})$. Assume that there exist some $\Theta \in \digamma$ and $k \in(0,1)$ so that

$$
\Theta\left(H\left([\mathcal{Q} \varphi]_{\alpha_{\mathcal{Q}}(\varphi)},[\mathcal{Q} \psi]_{\alpha_{\mathcal{Q}}(\psi)}\right)\right) \leq\left[\Theta\left(\rho\left(\begin{array}{c}
\varrho(\varphi, \psi), \varrho\left(\varphi,[\mathcal{Q} \varphi]_{\alpha_{\mathcal{Q}}(\varphi)}\right), \varrho\left(\psi,[\mathcal{Q} \psi]_{\alpha_{\mathcal{Q}}(\psi)}\right), \\
\varrho\left(\varphi,[\mathcal{Q} \psi]_{\alpha_{\mathcal{Q}}(\psi)}\right), \varrho\left(\psi,[\mathcal{Q} \varphi]_{\alpha_{\mathcal{Q}}(\varphi)}\right)
\end{array}\right)\right)\right]^{k}
$$

$\forall \varphi, \psi \in \mathcal{R}$ with $H\left([\mathcal{Q} \varphi]_{\alpha_{\mathcal{Q}}(\varphi)},[\mathcal{Q} \psi]_{\alpha_{\mathcal{Q}}(\psi)}\right)>0$. Then, there exists $\varphi^{*} \in \mathcal{R}$ such that $\varphi^{*} \in\left[\mathcal{Q}^{*}\right]_{\alpha_{\mathcal{Q}}\left(\varphi^{*}\right)}$.

\section{Consequences for Fuzzy Fixed Points}

Corollary 2. [7] Let $(\mathcal{R}, \varrho)$ be a complete metric space and let $\mathcal{Q}_{1}, \mathcal{Q}_{2}: \mathcal{R} \rightarrow(\mathcal{R})$ and for each $\varphi, \psi \in \mathcal{R}, \exists$ $\alpha_{\mathcal{Q}_{1}}(\varphi), \alpha_{\mathcal{Q}_{2}}(\psi) \in(0,1]$ such that $\left[\mathcal{Q}_{1} \varphi\right]_{\alpha_{\mathcal{Q}_{1}}(\varphi)},\left[\mathcal{Q}_{2} \psi\right]_{\alpha_{\mathcal{Q}_{2}}(\psi)} \in C B(\mathcal{R})$. Assume that there exist some $\Theta \in \digamma$ and $k \in(0,1)$ such that

$$
\Theta\left(H\left(\left[\mathcal{Q}_{1} \varphi\right]_{\alpha_{\mathcal{Q}_{1}}(\varphi)},\left[\mathcal{Q}_{2} \psi\right]_{\alpha_{\mathcal{Q}_{2}}(\psi)}\right)\right) \leq[\Theta(\varrho(\varphi, \psi))]^{k}
$$

$\forall \varphi, \psi \in \mathcal{R}$ with $H\left(\left[\mathcal{Q}_{1} \varphi\right]_{\alpha_{\mathcal{Q}_{1}}(\varphi)},\left[\mathcal{Q}_{2} \psi\right]_{\alpha_{\mathcal{Q}_{2}}(\psi)}\right)>0$. Then, there exists $\varphi^{*} \in \mathcal{R}$ such that $\varphi^{*} \in\left[\mathcal{Q}_{1} \varphi^{*}\right]_{\alpha_{\mathcal{Q}_{1}}\left(\varphi^{*}\right)} \cap$ $\left[\mathcal{Q}_{2} \varphi^{*}\right]_{\alpha_{\mathcal{Q}_{2}}\left(\varphi^{*}\right)}$

Proof. Consider $\rho \in \mathcal{P}$ given by $\rho\left(\varphi_{1}, \varphi_{2}, \varphi_{3}, \varphi_{4}, \varphi_{5}\right)=\varphi_{1}$. Then, the result follows from Theorem 2 .

Corollary 3. [7] Let $(\mathcal{R}, \varrho)$ be a complete metric space and let $\mathcal{Q}: \mathcal{R} \rightarrow(\mathcal{R})$ and for each $\varphi, \psi \in \mathcal{R}$, $\exists \alpha_{\mathcal{Q}}(\varphi), \alpha_{\mathcal{Q}}(\psi) \in(0,1]$ such that $[\mathcal{Q} \varphi]_{\alpha_{\mathcal{Q}}(\varphi)},[\mathcal{Q} \psi]_{\alpha_{\mathcal{Q}}(\psi)} \in C B(\mathcal{R})$. Assume that there exist some $\Theta \in \digamma$ and $k \in(0,1)$ such that

$$
\Theta\left(H\left([\mathcal{Q} \varphi]_{\alpha_{\mathcal{Q}}(\varphi)},[\mathcal{Q} \psi]_{\alpha_{\mathcal{Q}}(\psi)}\right)\right) \leq[\Theta(\varrho(\varphi, \psi))]^{k}
$$

$\forall \varphi, \psi \in \mathcal{R}$ with $H\left([\mathcal{Q} \varphi]_{\alpha_{\mathcal{Q}}(\varphi)},[\mathcal{Q} \psi]_{\alpha_{\mathcal{Q}}(\psi)}\right)>0$. Then, there exists $\varphi^{*} \in \mathcal{R}$ such that $\varphi^{*} \in\left[\mathcal{Q}^{*}\right]_{\alpha_{\mathcal{Q}}\left(\varphi^{*}\right)}$. 
Corollary 4. Let $(\mathcal{R}, \varrho)$ be a complete metric space and let $\mathcal{Q}_{1}, \mathcal{Q}_{2}: \mathcal{R} \rightarrow(\mathcal{R})$ and for each $\varphi, \psi \in \mathcal{R}$, $\exists \alpha_{\mathcal{Q}_{1}}(\varphi), \alpha_{\mathcal{Q}_{2}}(\psi) \in(0,1]$ such that $\left[\mathcal{Q}_{1} \varphi\right]_{\alpha_{\mathcal{Q}_{1}}(\varphi)},\left[\mathcal{Q}_{2} \psi\right]_{\alpha_{\mathcal{Q}_{2}}(\psi)} \in C B(\mathcal{R})$. Assume that there exist some $\Theta \in \digamma$ and $k \in(0,1)$ such that

$$
\Theta\left(H\left(\left[\mathcal{Q}_{1} \varphi\right]_{\alpha_{\mathcal{Q}_{1}}(\varphi)},\left[\mathcal{Q}_{2} \psi\right]_{\alpha_{\mathcal{Q}_{2}}(\psi)}\right)\right) \leq\left[\Theta\left(\varrho\left(\varphi,\left[\mathcal{Q}_{1} \varphi\right]_{\alpha_{\mathcal{Q}_{1}}(\varphi)}\right)+\varrho\left(\psi,\left[\mathcal{Q}_{2} \psi\right]_{\alpha_{\mathcal{Q}_{2}}(\psi)}\right)\right)\right]^{k}
$$

for all $\varphi, \psi \in \mathcal{R}$ with $H\left(\left[\mathcal{Q}_{1} \varphi\right]_{\mathcal{Q}_{\mathcal{Q}_{1}}(\varphi)},\left[\mathcal{Q}_{2} \psi\right]_{\mathcal{Q}_{\mathcal{Q}_{2}}(\psi)}\right)>0$. Then, there exists $\varphi^{*} \in \mathcal{R}$ such that $\varphi^{*} \in$ $\left[\mathcal{Q}_{1} \varphi^{*}\right]_{\alpha_{\mathcal{Q}_{1}}\left(\varphi^{*}\right)} \cap\left[\mathcal{Q}_{2} \varphi^{*}\right]_{\alpha_{\mathcal{Q}_{2}}\left(\varphi^{*}\right)} \cdot$

Proof. Consider $\rho \in \mathcal{P}$ given by $\rho\left(\varphi_{1}, \varphi_{2}, \varphi_{3}, \varphi_{4}, \varphi_{5}\right)=\varphi_{2}+\varphi_{3}$. Then, the result follows from Theorem 2.

Corollary 5. Let $(\mathcal{R}, \varrho)$ be a complete metric space and let $\mathcal{Q}_{1}, \mathcal{Q}_{2}: \mathcal{R} \rightarrow(\mathcal{R})$ and for each $\varphi, \psi \in \mathcal{R}$, $\exists \alpha_{\mathcal{Q}_{1}}(\varphi), \alpha_{\mathcal{Q}_{2}}(\psi) \in(0,1]$ such that $\left[\mathcal{Q}_{1} \varphi\right]_{\mathcal{Q}_{\mathcal{Q}_{1}}(\varphi)},\left[\mathcal{Q}_{2} \psi\right]_{\alpha_{\mathcal{Q}_{2}}(\psi)} \in C B(\mathcal{R})$. Assume that there exist some $\Theta \in \digamma$ and $k \in(0,1)$ such that

$$
\Theta\left(H\left(\left[\mathcal{Q}_{1} \varphi\right]_{\alpha_{\mathcal{Q}_{1}}(\varphi)},\left[\mathcal{Q}_{2} \psi\right]_{\alpha_{\mathcal{Q}_{2}}(\psi)}\right)\right) \leq\left[\Theta\left(\varrho\left(\varphi,\left[\mathcal{Q}_{2} \psi\right]_{\mathcal{Q}_{\mathcal{Q}_{2}}(\psi)}\right)+\varrho\left(\psi,\left[\mathcal{Q}_{1} \varphi\right]_{\alpha_{\mathcal{Q}_{1}}(\varphi)}\right)\right)\right]^{k}
$$

for all $\varphi, \psi \in \mathcal{R}$ with $H\left(\left[\mathcal{Q}_{1} \varphi\right]_{\alpha_{\mathcal{Q}_{1}}(\varphi)},\left[\mathcal{Q}_{2} \psi\right]_{\mathcal{Q}_{2}(\psi)}\right)>0$. Then, there exists $\varphi^{*} \in \mathcal{R}$ such that $\varphi^{*} \in$ $\left[\mathcal{Q}_{1} \varphi^{*}\right]_{\alpha_{\mathcal{Q}_{1}}\left(\varphi^{*}\right)} \cap\left[\mathcal{Q}_{2} \varphi^{*}\right]_{\alpha_{\mathcal{Q}_{2}}\left(\varphi^{*}\right)} \cdot$

Proof. Consider $\rho \in \mathcal{P}$ given by $\rho\left(\varphi_{1}, \varphi_{2}, \varphi_{3}, \varphi_{4}, \varphi_{5}\right)=\varphi_{4}+\varphi_{5}$. Then, the result follows from Theorem 2.

Corollary 6. Let $(\mathcal{R}, \varrho)$ be a complete metric space and let $\mathcal{Q}_{1}, \mathcal{Q}_{2}: \mathcal{R} \rightarrow(\mathcal{R})$ and for each $\varphi, \psi \in \mathcal{R}$, $\exists \alpha_{\mathcal{Q}_{1}}(\varphi), \alpha_{\mathcal{Q}_{2}}(\psi) \in(0,1]$ such that $\left[\mathcal{Q}_{1} \varphi\right]_{\alpha_{\mathcal{Q}_{1}}(\varphi)},\left[\mathcal{Q}_{2} \psi\right]_{\alpha_{\mathcal{Q}_{2}}(\psi)} \in C B(\mathcal{R})$. Assume that there exist some $\Theta \in \digamma$ and $k \in(0,1)$ and non-negative real numbers $\alpha, \beta, \gamma$ with $\alpha+\beta+\gamma \leq 1$ such that

$$
\Theta\left(H\left(\left[\mathcal{Q}_{1} \varphi\right]_{\alpha_{\mathcal{Q}_{1}}(\varphi)},\left[\mathcal{Q}_{2} \psi\right]_{\alpha_{\mathcal{Q}_{2}}(\psi)}\right)\right) \leq\left[\Theta\left(\alpha \varrho(\varphi, \psi)+\beta \varrho\left(\varphi,\left[\mathcal{Q}_{1} \varphi\right]_{\alpha_{\mathcal{Q}_{1}}(\varphi)}\right)+\gamma \varrho\left(\psi,\left[\mathcal{Q}_{2} \psi\right]_{\alpha_{\mathcal{Q}_{2}}(\psi)}\right)\right)\right]^{k}
$$

for all $\varphi, \psi \in \mathcal{R}$ with $H\left(\left[\mathcal{Q}_{1} \varphi\right]_{\alpha_{\mathcal{Q}_{1}}(\varphi)},\left[\mathcal{Q}_{2} \psi\right]_{\alpha_{\mathcal{Q}_{2}}(\psi)}\right)>0$. Then, there exists $\varphi^{*} \in \mathcal{R}$ such that $\varphi^{*} \in$ $\left[\mathcal{Q}_{1} \varphi^{*}\right]_{\alpha_{\mathcal{Q}_{1}}\left(\varphi^{*}\right)} \cap\left[\mathcal{Q}_{2} \varphi^{*}\right]_{\alpha_{\mathcal{Q}_{2}}\left(\varphi^{*}\right)} \cdot$

Proof. Consider $\rho \in \mathcal{P}$ given by $\rho\left(\varphi_{1}, \varphi_{2}, \varphi_{3}, \varphi_{4}, \varphi_{5}\right)=\alpha \varphi_{1}+\beta \varphi_{2}+\gamma \varphi_{3}$. Then, the result follows from Theorem 2.

Corollary 7. Let $(\mathcal{R}, \varrho)$ be a complete metric space and let $\mathcal{Q}_{1}, \mathcal{Q}_{2}: \mathcal{R} \rightarrow(\mathcal{R})$ and for each $\varphi, \psi \in \mathcal{R}$, $\exists \alpha_{\mathcal{Q}_{1}}(\varphi), \alpha_{\mathcal{Q}_{2}}(\psi) \in(0,1]$ such that $\left[\mathcal{Q}_{1} \varphi\right]_{\alpha_{\mathcal{Q}_{1}}(\varphi)},\left[\mathcal{Q}_{2} \psi\right]_{\alpha_{\mathcal{Q}_{2}}(\psi)} \in C B(\mathcal{R})$. Assume that there exist some $\Theta \in \digamma$ and $k \in(0,1)$ and non-negative real number $\alpha \in(0,1]$ and $L \geq 0$ such that

$$
\Theta\left(H\left(\left[\mathcal{Q}_{1} \varphi\right]_{\alpha_{\mathcal{Q}_{1}}(\varphi)},\left[\mathcal{Q}_{2} \psi\right]_{\alpha_{\mathcal{Q}_{2}}(\psi)}\right)\right) \leq\left[\Theta\left(\alpha \varrho(\varphi, \psi)+L \varrho\left(\psi,\left[\mathcal{Q}_{1} \varphi\right]_{\alpha_{\mathcal{Q}_{1}}(\varphi)}\right)\right)\right]^{k}
$$

for all $\varphi, \psi \in \mathcal{R}$ with $H\left(\left[\mathcal{Q}_{1} \varphi\right]_{\alpha_{\mathcal{Q}_{1}}(\varphi)},\left[\mathcal{Q}_{2} \psi\right]_{\alpha_{\mathcal{Q}_{2}}(\psi)}\right)>0$. Then, there exists $\varphi^{*} \in \mathcal{R}$ such that $\varphi^{*} \in$ $\left[\mathcal{Q}_{1} \varphi^{*}\right]_{\alpha_{\mathcal{Q}_{1}}\left(\varphi^{*}\right)} \cap\left[\mathcal{Q}_{2} \varphi^{*}\right]_{\alpha_{\mathcal{Q}_{2}}\left(\varphi^{*}\right)} \cdot$ 
Proof. Consider $\rho \in \mathcal{P}$ given by $\rho\left(\varphi_{1}, \varphi_{2}, \varphi_{3}, \varphi_{4}, \varphi_{5}\right)=\alpha \varphi_{1}+L \varphi_{5}$. Then, the result follows from Theorem 2.

Corollary 8. Let $(\mathcal{R}, \varrho)$ be a complete metric space and let $\mathcal{Q}_{1}, \mathcal{Q}_{2}: \mathcal{R} \rightarrow(\mathcal{R})$ and for each $\varphi, \psi \in \mathcal{R}$, $\exists \alpha_{\mathcal{Q}_{1}}(\varphi), \alpha_{\mathcal{Q}_{2}}(\psi) \in(0,1]$ such that $\left[\mathcal{Q}_{1} \varphi\right]_{\alpha_{\mathcal{Q}_{1}}(\varphi)},\left[\mathcal{Q}_{2} \psi\right]_{\alpha_{\mathcal{Q}_{2}}(\psi)} \in C B(\mathcal{R})$. Assume that there exist some $\Theta \in \digamma$ and $k \in(0,1)$ and non-negative real numbers $\alpha, \beta, \gamma, \delta, L$ with $\alpha+\beta+\gamma+\delta+2 L \leq 1$ such that

$$
\Theta\left(H\left(\left[\mathcal{Q}_{1} \varphi\right]_{\alpha_{\mathcal{Q}_{1}}(\varphi)},\left[\mathcal{Q}_{2} \psi\right]_{\alpha_{\mathcal{Q}_{2}}(\psi)}\right)\right) \leq\left[\Theta\left(\begin{array}{c}
\alpha \varrho(\varphi, \psi)+\beta \varrho\left(\varphi,\left[\mathcal{Q}_{1} \varphi\right]_{\alpha_{\mathcal{Q}_{1}}(\varphi)}\right)+\gamma \varrho\left(\psi,\left[\mathcal{Q}_{2} \psi\right]_{\alpha_{\mathcal{Q}_{2}}(\psi)}\right) \\
+\delta \varrho\left(\varphi,\left[\mathcal{Q}_{2} \psi\right]_{\alpha_{\mathcal{Q}_{2}}(\psi)}\right)+L \varrho\left(\psi,\left[\mathcal{Q}_{1} \varphi\right]_{\alpha_{\mathcal{Q}_{1}}(\varphi)}\right)
\end{array}\right)\right]^{k}
$$

for all $\varphi, \psi \in \mathcal{R}$ with $H\left(\left[\mathcal{Q}_{1} \varphi\right]_{\mathcal{Q}_{\mathcal{Q}_{1}}(\varphi)},\left[\mathcal{Q}_{2} \psi\right]_{\alpha_{\mathcal{Q}_{2}}(\psi)}\right)>0$. Then, there exists $\varphi^{*} \in \mathcal{R}$ such that $\varphi^{*} \in$ $\left[\mathcal{Q}_{1} \varphi^{*}\right]_{\alpha_{\mathcal{Q}_{1}}\left(\varphi^{*}\right)} \cap\left[\mathcal{Q}_{2} \varphi^{*}\right]_{\alpha_{\mathcal{Q}_{2}}\left(\varphi^{*}\right)} \cdot$

Proof. Consider $\rho \in \mathcal{P}$ given by $\rho\left(\varphi_{1}, \varphi_{2}, \varphi_{3}, \varphi_{4}, \varphi_{5}\right)=\alpha \varphi_{1}+\beta \varphi_{2}+\gamma \varphi_{3}+\delta \varphi_{4}+L \varphi_{5}$. Then, the result follows from Theorem 2.

Corollary 9. [3] Let $(\mathcal{R}, \varrho)$ be a complete metric space and let $\mathcal{Q}_{1}, \mathcal{Q}_{2}: \mathcal{R} \rightarrow(\mathcal{R})$ and for each $\varphi, \psi \in \mathcal{R}, \exists$ $\alpha_{\mathcal{Q}_{1}}(\varphi), \alpha_{\mathcal{Q}_{2}}(\psi) \in(0,1]$ such that $\left[\mathcal{Q}_{1} \varphi\right]_{\alpha_{\mathcal{Q}_{1}}(\varphi)},\left[\mathcal{Q}_{2} \psi\right]_{\alpha_{\mathcal{Q}_{2}}(\psi)} \in C B(\mathcal{R})$. Assume that there exist some $\Theta \in \digamma$ and $k \in(0,1)$ such that

$$
\Theta\left(H\left(\left[\mathcal{Q}_{1} \varphi\right]_{\mathcal{Q}_{\mathcal{Q}_{1}}(\varphi)},\left[\mathcal{Q}_{2} \psi\right]_{\mathcal{Q}_{\mathcal{Q}_{2}}(\psi)}\right)\right) \leq\left[\Theta\left(\max \left\{\begin{array}{c}
\varrho(\varphi, \psi), \varrho\left(\varphi,\left[\mathcal{Q}_{1} \varphi\right]_{\alpha_{\mathcal{Q}_{1}}(\varphi)}\right), \varrho\left(\psi,\left[\mathcal{Q}_{2} \psi\right]_{\alpha_{\mathcal{Q}_{2}}(\psi)}\right), \\
\frac{\varrho\left(\varphi,\left[\mathcal{Q}_{2} \psi\right]_{\mathcal{Q}_{\mathcal{Q}_{2}}(\psi)}\right)+\varrho\left(\psi,\left[\mathcal{Q}_{1} \varphi\right]_{\mathcal{Q}_{\mathcal{Q}_{1}}(\varphi)}\right)}{2}
\end{array}\right\}\right)\right]^{k}
$$

for all $\varphi, \psi \in \mathcal{R}$ with $H\left(\left[\mathcal{Q}_{1} \varphi\right]_{\alpha_{\mathcal{Q}_{1}}(\varphi)},\left[\mathcal{Q}_{2} \psi\right]_{\alpha_{\mathcal{Q}_{2}}(\psi)}\right)>0$. Then, there exists $\varphi^{*} \in \mathcal{R}$ such that $\varphi^{*} \in$ $\left[\mathcal{Q}_{1} \varphi^{*}\right]_{\alpha_{\mathcal{Q}_{1}}\left(\varphi^{*}\right)} \cap\left[\mathcal{Q}_{2} \varphi^{*}\right]_{\alpha_{\mathcal{Q}_{2}}\left(\varphi^{*}\right)} \cdot$

Proof. Consider $\rho \in \mathcal{P}$ given by $\rho\left(\varphi_{1}, \varphi_{2}, \varphi_{3}, \varphi_{4}, \varphi_{5}\right)=\max \left\{\varphi_{1}, \varphi_{2}, \varphi_{3}, \frac{\varphi_{4}+\varphi_{5}}{2}\right\}$ Then, the result follows from Theorem 2.

Corollary 10. Let $(\mathcal{R}, \varrho)$ be a complete metric space and let $\mathcal{Q}_{1}, \mathcal{Q}_{2}: \mathcal{R} \rightarrow(\mathcal{R})$ and for each $\varphi, \psi \in \mathcal{R}, \exists$ $\alpha_{\mathcal{Q}_{1}}(\varphi), \alpha_{\mathcal{Q}_{2}}(\psi) \in(0,1]$ such that $\left[\mathcal{Q}_{1} \varphi\right]_{\alpha_{\mathcal{Q}_{1}}(\varphi)},\left[\mathcal{Q}_{2} \psi\right]_{\alpha_{\mathcal{Q}_{2}}(\psi)} \in C B(\mathcal{R})$. Assume that there exist some $\Theta \in \digamma$ and $k \in(0,1)$ such that

$$
\Theta\left(H\left(\left[\mathcal{Q}_{1} \varphi\right]_{\alpha_{\mathcal{Q}_{1}}(\varphi)},\left[\mathcal{Q}_{2} \psi\right]_{\alpha_{\mathcal{Q}_{2}}(\psi)}\right)\right) \leq\left[\Theta\left(\max \left\{\begin{array}{c}
\varrho(\varphi, \psi), \varrho\left(\varphi,\left[\mathcal{Q}_{1} \varphi\right]_{\alpha_{\mathcal{Q}_{1}}(\varphi)}\right), \varrho\left(\psi,\left[\mathcal{Q}_{2} \psi\right]_{\alpha_{\mathcal{Q}_{2}}(\psi)}\right), \\
\varrho\left(\varphi,\left[\mathcal{Q}_{2} \psi\right]_{\alpha_{\mathcal{Q}_{2}}(\psi)}\right), \varrho\left(\psi,\left[\mathcal{Q}_{1} \varphi\right]_{\alpha_{\mathcal{Q}_{1}}(\varphi)}\right)
\end{array}\right\}\right)\right]^{k}
$$

for all $\varphi, \psi \in \mathcal{R}$ with $H\left(\left[\mathcal{Q}_{1} \varphi\right]_{\alpha_{\mathcal{Q}_{1}}(\varphi)},\left[\mathcal{Q}_{2} \psi\right]_{\alpha_{\mathcal{Q}_{2}}(\psi)}\right)>0$. Then, there exists $\varphi^{*} \in \mathcal{R}$ such that $\varphi^{*} \in$ $\left[\mathcal{Q}_{1} \varphi^{*}\right]_{\alpha_{\mathcal{Q}_{1}}\left(\varphi^{*}\right)} \cap\left[\mathcal{Q}_{2} \varphi^{*}\right]_{\alpha_{\mathcal{Q}_{2}}\left(\varphi^{*}\right)} \cdot$

Proof. Consider $\rho \in \mathcal{P}$ given by $\rho\left(\varphi_{1}, \varphi_{2}, \varphi_{3}, \varphi_{4}, \varphi_{5}\right)=\max \left\{\varphi_{1}, \varphi_{2}, \varphi_{3}, \varphi_{4}, \varphi_{5}\right\}$. Then, the result follows from Theorem 2. 
Corollary 11. Let $(\mathcal{R}, \varrho)$ be a complete metric space and let $\mathcal{Q}_{1}, \mathcal{Q}_{2}: \mathcal{R} \rightarrow(\mathcal{R})$ and for each $\varphi, \psi \in \mathcal{R}, \exists$ $\alpha_{\mathcal{Q}_{1}}(\varphi), \alpha_{\mathcal{Q}_{2}}(\psi) \in(0,1]$ such that $\left[\mathcal{Q}_{1} \varphi\right]_{\alpha_{\mathcal{Q}_{1}}(\varphi)},\left[\mathcal{Q}_{2} \psi\right]_{\alpha_{\mathcal{Q}_{2}}(\psi)} \in C B(\mathcal{R})$. Assume that there exist some $\Theta \in \digamma$ and $k \in(0,1)$ such that

$$
\Theta\left(H\left([\mathcal{Q} \varphi]_{\alpha_{\mathcal{Q}}(\varphi)},[\mathcal{Q} \psi]_{\alpha_{\mathcal{Q}}(\psi)}\right)\right) \leq\left[\Theta\left(\max \left\{\begin{array}{c}
\varrho(\varphi, \psi), \varrho\left(\varphi,[\mathcal{Q} \varphi]_{\alpha_{\mathcal{Q}}(\varphi)}\right), \varrho\left(\psi,[\mathcal{Q} \psi]_{\alpha_{\mathcal{Q}}(\psi)}\right), \\
\varrho\left(\varphi,[\mathcal{Q} \psi]_{\alpha_{\mathcal{Q}}(\psi)}\right), \varrho\left(\psi,[\mathcal{Q} \varphi]_{\alpha_{\mathcal{Q}}(\varphi)}\right)
\end{array}\right\}\right)\right]^{k}
$$

for all $\varphi, \psi \in \mathcal{R}$ with $H\left([\mathcal{Q} \varphi]_{\mathcal{Q}_{\mathcal{Q}}(\varphi)},[\mathcal{Q} \psi]_{\mathcal{Q}_{\mathcal{Q}}(\psi)}\right)>0$. Then, there exists $\varphi^{*} \in \mathcal{R}$ such that $\varphi^{*} \in\left[\mathcal{Q}^{*}\right]_{\alpha_{\mathcal{Q}}\left(\varphi^{*}\right)}$.

Corollary 12. Let $(\mathcal{R}, \varrho)$ be a complete metric space and let $\mathcal{Q}_{1}, \mathcal{Q}_{2}: \mathcal{R} \rightarrow(\mathcal{R})$ and for each $\varphi, \psi \in \mathcal{R}, \exists$ $\alpha_{\mathcal{Q}_{1}}(\varphi), \alpha_{\mathcal{Q}_{2}}(\psi) \in(0,1]$ such that $\left[\mathcal{Q}_{1} \varphi\right]_{\alpha_{\mathcal{Q}_{1}}(\varphi)},\left[\mathcal{Q}_{2} \psi\right]_{\alpha_{\mathcal{Q}_{2}}(\psi)} \in C B(\mathcal{R})$. Assume that there exist some $\Theta \in \digamma$ and $k \in(0,1)$ such that

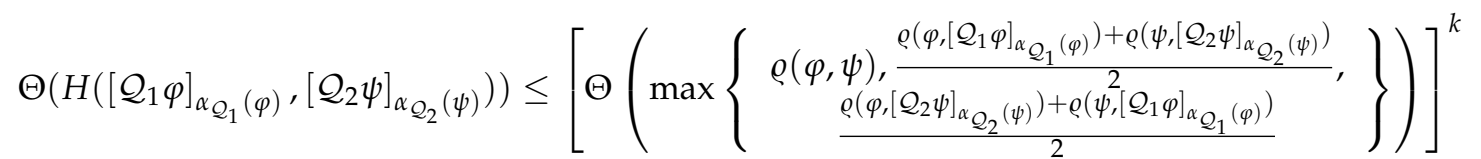

for all $\varphi, \psi \in \mathcal{R}$ with $H\left(\left[\mathcal{Q}_{1} \varphi\right]_{\alpha_{\mathcal{Q}_{1}}(\varphi)},\left[\mathcal{Q}_{2} \psi\right]_{\alpha_{\mathcal{Q}_{2}}(\psi)}\right)>0$. Then, there exists $\varphi^{*} \in \mathcal{R}$ such that $\varphi^{*} \in$ $\left[\mathcal{Q}_{1} \varphi^{*}\right]_{\alpha_{\mathcal{Q}_{1}}\left(\varphi^{*}\right)} \cap\left[\mathcal{Q}_{2} \varphi^{*}\right]_{\alpha_{\mathcal{Q}_{2}}\left(\varphi^{*}\right)} \cdot$

Proof. Consider $\rho \in \mathcal{P}$ given by $\rho\left(\varphi_{1}, \varphi_{2}, \varphi_{3}, \varphi_{4}, \varphi_{5}\right)=\max \left\{\varphi_{1} \frac{\varphi_{2}+\varphi_{3}}{2}, \frac{\varphi_{4}+\varphi_{5}}{2}\right\}$. Then, the result follows from Theorem 2.

\section{Consequences for Multivalued Mappings}

Fixed point theorems for multivalued mappings can be derived from fuzzy fixed point theorems in this way.

Theorem 3. Let $(\mathcal{R}, \varrho)$ be a complete metric space and let $F, G: \mathcal{R} \rightarrow C B(\mathcal{R})$. Assume that there exist some $\Theta \in \digamma$ and $k \in(0,1)$ such that

$$
H(F \varphi, G \psi)>0 \Longrightarrow \Theta(H(F \varphi, G \psi)) \leq\left[\Theta\left(\rho\left(\begin{array}{c}
\varrho(\varphi, \psi), \varrho(\varphi, F \varphi), \varrho(\psi, G \psi), \\
\varrho(\varphi, G \psi), \varrho(\psi, F \varphi)
\end{array}\right)\right)\right]^{k}
$$

$\forall \varphi, \psi \in \mathcal{R}$. Then, F and $G$ a have common fixed point.

Proof. Define $\alpha: \mathcal{R} \rightarrow(0,1]$ and $\mathcal{Q}_{1}, \mathcal{Q}_{2}: \mathcal{R} \rightarrow(\mathcal{R})$ by

$$
\mathcal{Q}_{1}(\varphi)(t)=\left\{\begin{array}{c}
\alpha(\varphi), \text { if } t \in F \varphi \\
0, \text { if } t \notin F \varphi
\end{array}\right.
$$

and

$$
\mathcal{Q}_{2}(\varphi)(t)=\left\{\begin{array}{c}
\alpha(\varphi), \text { if } t \in G \varphi \\
0, \text { if } t \notin G \varphi
\end{array}\right.
$$

Then,

$$
\left[\mathcal{Q}_{1} \varphi\right]_{\alpha(\varphi)}=\left\{t: \mathcal{Q}_{1}(\varphi)(t) \geq \alpha(\varphi)\right\}=F \varphi \text { and } \quad\left[\mathcal{Q}_{2} \varphi\right]_{\alpha(\varphi)}=\left\{t: \mathcal{Q}_{2}(\varphi)(t) \geq \alpha(\varphi)\right\}=G \varphi
$$


Thus, Theorem 2 can be applied to obtain $\varphi^{*} \in \mathcal{R}$ such that $\varphi^{*} \in\left[\mathcal{Q}_{1} \varphi^{*}\right]_{\alpha_{\mathcal{Q}_{1}}\left(\varphi^{*}\right)} \cap\left[\mathcal{Q}_{2} \varphi^{*}\right]_{\mathcal{Q}_{2}\left(\varphi^{*}\right)}=$ $F \varphi^{*} \cap G \varphi^{*}$.

The main result of Isik [11] can be deduced by taking one mapping in Theorem 3 .

Corollary 13. [11] Let $(\mathcal{R}, \varrho)$ be a complete metric space and let $G: \mathcal{R} \rightarrow C B(\mathcal{R})$. Assume that there exist some $\Theta \in \digamma$ and $k \in(0,1)$ such that

$$
H(G \varphi, G \psi)>0 \Longrightarrow \Theta(H(G \varphi, G \psi)) \leq\left[\Theta\left(\rho\left(\begin{array}{c}
\varrho(\varphi, \psi), \varrho(\varphi, G \varphi), \varrho(\psi, G \psi), \\
\varrho(\varphi, G \psi), \varrho(\psi, G \varphi)
\end{array}\right)\right)\right]^{k}
$$

for all $\varphi, \psi \in \mathcal{R}$. Then, there exists $\varphi^{*} \in \mathcal{R}$ such that $\varphi^{*} \in G \varphi^{*}$.

We derive the main result of Vetro [6], which is itself a $\Theta$-generalization of Nadler's [9] fixed point theorem from Theorem 3.

Corollary 14. [6] Let $(\mathcal{R}, \varrho)$ be a complete metric space and let $G: \mathcal{R} \rightarrow C B(\mathcal{R})$. Assume that there exist some $\Theta \in \digamma$ and $k \in(0,1)$ such that

$$
H(G \varphi, G \psi)>0 \Longrightarrow \Theta(H(G \varphi, G \psi)) \leq[\Theta(\varrho(\varphi, \psi))]^{k}
$$

for all $\varphi, \psi \in \mathcal{R}$. Then, there exists $\varphi^{*} \in \mathcal{R}$ such that $\varphi^{*} \in G \varphi^{*}$.

Proof. Consider $\rho \in \mathcal{P}$ given by $\rho\left(\varphi_{1}, \varphi_{2}, \varphi_{3}, \varphi_{4}, \varphi_{5}\right)=\varphi_{1}$ and $F=G$. Then, the result follows from Theorem 3.

The following result is a $\Theta$-generalization of Kannan [21] fixed point theorem for multivalued mapping.

Corollary 15. Let $(\mathcal{R}, \varrho)$ be a complete metric space and let $G: \mathcal{R} \rightarrow C B(\mathcal{R})$. Assume that there exist some $\Theta \in \digamma$ and $k \in(0,1)$ such that

$$
H(G \varphi, G \psi)>0 \Longrightarrow H(G \varphi, G \psi)>0 \Longrightarrow \Theta(H(G \varphi, G \psi)) \leq[\Theta(\varrho(\varphi, G \varphi)+\varrho(\psi, G \psi))]^{k}
$$

for all $\varphi, \psi \in \mathcal{R}$. Then, there exists $\varphi^{*} \in \mathcal{R}$ such that $\varphi^{*} \in G \varphi^{*}$.

Proof. Consider $\rho \in \mathcal{P}$ given by $\rho\left(\varphi_{1}, \varphi_{2}, \varphi_{3}, \varphi_{4}, \varphi_{5}\right)=\varphi_{2}+\varphi_{3}$ and $F=G$. Then, the result follows from Theorem 3.

The following result is a $\Theta$-generalization of Chatterjea [22] fixed point theorem for multivalued mapping.

Corollary 16. Let $(\mathcal{R}, \varrho)$ be a complete metric space and let $G: \mathcal{R} \rightarrow C B(\mathcal{R})$. Assume that there exist some $\Theta \in \digamma$ and $k \in(0,1)$ such that

$$
H(G \varphi, G \psi)>0 \Longrightarrow \Theta(H(G \varphi, G \psi)) \leq[\Theta(\varrho(\varphi, G \psi)+\varrho(\psi, G \varphi))]^{k}
$$

for all $\varphi, \psi \in \mathcal{R}$. Then, there exists $\varphi^{*} \in \mathcal{R}$ such that $\varphi^{*} \in G \varphi^{*}$. 
Proof. Consider $\rho \in \mathcal{P}$ given by $\rho\left(\varphi_{1}, \varphi_{2}, \varphi_{3}, \varphi_{4}, \varphi_{5}\right)=\varphi_{4}+\varphi_{5}$ and $F=G$. Then, the result follows from Theorem 3.

The following result is a $\Theta$-generalization of Reich $[23,24]$ fixed point theorem for multivalued mapping.

Corollary 17. Let $(\mathcal{R}, \varrho)$ be a complete metric space and let $G: \mathcal{R} \rightarrow C B(\mathcal{R})$. Assume that there exist some $\Theta \in \digamma$ and $k \in(0,1)$ and non-negative real numbers $\alpha, \beta, \gamma$ with $\alpha+\beta+\gamma \leq 1$ such that

$$
H(G \varphi, G \psi)>0 \Longrightarrow \Theta(H(G \varphi, G \psi)) \leq[\Theta(\alpha \varrho(\varphi, \psi)+\beta \varrho(\varphi, G \varphi)+\gamma \varrho(\psi, G \psi))]^{k}
$$

for all $\varphi, \psi \in \mathcal{R}$. Then, there exists $\varphi^{*} \in \mathcal{R}$ such that $\varphi^{*} \in G \varphi^{*}$.

Proof. Consider $\rho \in \mathcal{P}$ given by $\rho\left(\varphi_{1}, \varphi_{2}, \varphi_{3}, \varphi_{4}, \varphi_{5}\right)=\alpha \varphi_{1}+\beta \varphi_{2}+\gamma \varphi_{3}$ and $F=G$. Then, the result follows from Theorem 3.

The following result is a $\Theta$-generalization of Berinde's [25] fixed point theorem for multivalued mapping.

Corollary 18. Let $(\mathcal{R}, \varrho)$ be a complete metric space and let $G: \mathcal{R} \rightarrow C B(\mathcal{R})$. Assume that there exist some $\Theta \in \digamma$ and $k \in(0,1)$ and non-negative real number $\alpha \in(0,1]$ and $L \geq 0$ such that

$$
H(G \varphi, G \psi)>0 \Longrightarrow \Theta(H(G \varphi, G \psi)) \leq[\Theta(\alpha \varrho(\varphi, \psi)+L \varrho(\psi, G \varphi))]^{k}
$$

for all $\varphi, \psi \in \mathcal{R}$. Then, there exists $\varphi^{*} \in \mathcal{R}$ such that $\varphi^{*} \in G \varphi^{*}$.

Proof. Consider $\rho \in \mathcal{P}$ given by $\rho\left(\varphi_{1}, \varphi_{2}, \varphi_{3}, \varphi_{4}, \varphi_{5}\right)=\alpha \varphi_{1}+L \varphi_{5}$ and $F=G$. Then, the result follows from Theorem 3.

The following result is a $\Theta$-generalization of Hardy and Rogers' [26] fixed point theorem for multivalued mapping.

Corollary 19. Let $(\mathcal{R}, \varrho)$ be a complete metric space and let $G: \mathcal{R} \rightarrow C B(\mathcal{R})$. Assume that there exist some $\Theta \in \digamma$ and $k \in(0,1)$ and non-negative real numbers $\alpha, \beta, \gamma, \delta$ and $L$ with $\alpha+\beta+\gamma+\delta+2 L \leq 1$ such that

$$
H(G \varphi, G \psi)>0 \Longrightarrow \Theta(H(G \varphi, G \psi)) \leq\left[\Theta\left(\begin{array}{c}
\alpha \varrho(\varphi, \psi)+\beta \varrho(\varphi, G \varphi)+\gamma \varrho(\psi, G \psi) \\
+\delta \varrho(\varphi, G \psi)+L \varrho(\psi, G \varphi)
\end{array}\right)\right]^{k}
$$

for all $\varphi, \psi \in \mathcal{R}$. Then, there exists $\varphi^{*} \in \mathcal{R}$ such that $\varphi^{*} \in G \varphi^{*}$.

Proof. Consider $\rho \in \mathcal{P}$ given by $\rho\left(\varphi_{1}, \varphi_{2}, \varphi_{3}, \varphi_{4}, \varphi_{5}\right)=\alpha \varphi_{1}+\beta \varphi_{2}+\gamma \varphi_{3}+\delta \varphi_{4}+L \varphi_{5}$ and $F=G$. Then, the result follows from Theorem 3 .

The following result is a $\Theta$-generalization of Ćirić [27] fixed point theorem for multivalued mapping.

Corollary 20. Let $(\mathcal{R}, \varrho)$ be a complete metric space and let $F, G: \mathcal{R} \rightarrow C B(\mathcal{R})$. Assume that there exist some $\Theta \in \digamma$ and $k \in(0,1)$ such that

$$
H(F \varphi, G \psi)>0 \Longrightarrow \Theta(H(F \varphi, G \psi)) \leq\left[\Theta\left(\max \left\{\begin{array}{c}
\varrho(\varphi, \psi), \varrho(\varphi, F \varphi), \varrho(\psi, G \psi), \\
\frac{\rho(\varphi, G \psi)+\varrho(\psi, F \varphi)}{2}
\end{array}\right\}\right)\right]^{k}
$$


for all $\varphi, \psi \in \mathcal{R}$. Then, $F$ and $G$ have common fixed point.

Proof. Consider $\rho \in \mathcal{P}$ given by $\rho\left(\varphi_{1}, \varphi_{2}, \varphi_{3}, \varphi_{4}, \varphi_{5}\right)=\max \left\{\varphi_{1}, \varphi_{2}, \varphi_{3}, \frac{\varphi_{4}+\varphi_{5}}{2}\right\}$ Then, the result follows from Theorem 3.

Corollary 21. Let $(\mathcal{R}, \varrho)$ be a complete metric space and let $G: \mathcal{R} \rightarrow C B(\mathcal{R})$. Assume that there exist some $\Theta \in \digamma$ and $k \in(0,1)$ such that

$$
H(G \varphi, G \psi)>0 \Longrightarrow \Theta(H(G \varphi, G \psi)) \leq\left[\Theta\left(\max \left\{\begin{array}{c}
\varrho(\varphi, \psi), \varrho(\varphi, G \varphi), \varrho(\psi, G \psi), \\
\frac{\varrho(\varphi, G \psi)+\varrho(\psi, G \varphi)}{2}
\end{array}\right\}\right)\right]^{k}
$$

for all $\varphi, \psi \in \mathcal{R}$. Then, there exists $\varphi^{*} \in \mathcal{R}$ such that $\varphi^{*} \in G \varphi^{*}$.

The following result is a $\Theta$-generalization of Ćirić [28] fixed point theorem for multivalued mapping.

Corollary 22. Let $(\mathcal{R}, \varrho)$ be a complete metric space and let $F, G: \mathcal{R} \rightarrow C B(\mathcal{R})$. Assume that there exist some $\Theta \in \digamma$ and $k \in(0,1)$ such that

$$
H(F \varphi, G \psi)>0 \Longrightarrow \Theta(H(F \varphi, G \psi)) \leq\left[\Theta\left(\max \left\{\begin{array}{c}
\varrho(\varphi, \psi), \varrho(\varphi, F \varphi), \varrho(\psi, G \psi), \\
\varrho(\varphi, G \psi), \varrho(\psi, F \varphi)
\end{array}\right\}\right)\right]^{k}
$$

for all $\varphi, \psi \in \mathcal{R}$. Then, there exists $\varphi^{*} \in \mathcal{R}$ such that $\varphi^{*} \in F \varphi^{*} \cap G \varphi^{*}$.

Proof. Consider $\rho \in \mathcal{P}$ given by $\rho\left(\varphi_{1}, \varphi_{2}, \varphi_{3}, \varphi_{4}, \varphi_{5}\right)=\max \left\{\varphi_{1}, \varphi_{2}, \varphi_{3}, \varphi_{4}, \varphi_{5}\right\}$. Then, the result follows from Theorem 3.

The following result is a $\Theta$-generalization of Ćirićs $[27,28]$ fixed point theorem for multivalued mapping.

Corollary 23. Let $(\mathcal{R}, \varrho)$ be a complete metric space and let $G: \mathcal{R} \rightarrow C B(\mathcal{R})$. Assume that there exist some $\Theta \in \digamma$ and $k \in(0,1)$ such that

$$
H(G \varphi, G \psi)>0 \Longrightarrow \Theta(H(G \varphi, G \psi)) \leq\left[\Theta\left(\max \left\{\begin{array}{c}
\varrho(\varphi, \psi), \varrho(\varphi, G \varphi), \varrho(\psi, G \psi), \\
\varrho(\varphi, G \psi), \varrho(\psi, G \varphi)
\end{array}\right\}\right)\right]^{k}
$$

$\forall \varphi, \psi \in \mathcal{R}$. Then, $F$ and $G$ have common fixed point.

Corollary 24. Let $(\mathcal{R}, \varrho)$ be a complete metric space and let $F, G: \mathcal{R} \rightarrow C B(\mathcal{R})$. Assume that there exist some $\Theta \in \digamma$ and $k \in(0,1)$ such that

$$
\Theta(H(F \varphi, G \psi)) \leq\left[\Theta\left(\max \left\{\varrho(\varphi, \psi), \frac{\varrho(\varphi, F \varphi)+\varrho(\psi, G \psi)}{2}, \frac{\varrho(\varphi, G \psi)+\varrho(\psi, G \varphi)}{2}\right\}\right)\right]
$$

for all $\varphi, \psi \in \mathcal{R}$ with $H(F \varphi, G \psi)>0$. Then, there exists $\varphi^{*} \in \mathcal{R}$ such that $\varphi^{*} \in F \varphi^{*} \cap G \varphi^{*}$.

Proof. Consider $\rho \in \mathcal{P}$ given by $\rho\left(\varphi_{1}, \varphi_{2}, \varphi_{3}, \varphi_{4}, \varphi_{5}\right)=\max \left\{\varphi_{1}, \frac{\varphi_{2}+\varphi_{3}}{2}, \frac{\varphi_{4}+\varphi_{5}}{2}\right\}$ Then, the result follows from Theorem 3. 


\section{Applications}

The aim of this section is to apply the established results to obtain the existence of solutions for a certain Fredholm integral inclusion. Consider the following integral inclusion of Fredholm type,

$$
\varphi(t) \in\left[f(t)+\int_{0}^{1} K(t, s, \varphi(s)) \varrho s\right]
$$

for $t \in[0,1]$, where $f \in C[0,1]$ is a given real-valued function and $K:[0,1] \times[0,1] \times \mathbb{R} \rightarrow K_{c v}(\mathbb{R})$ a given multivalued operator, where $K_{c v}$ denotes the family of non-empty compact and convex subsets of $\mathbb{R}$ and $\varphi \in C[0,1]$ is the unknown function.

Now, consider the metric $\varrho$ on $C[0,1]$ defined by

$$
\varrho(\varphi, \psi)=\left(\max _{t \in[0,1]}|\varphi(t)-\psi(t)|\right)=\max _{t \in[0,1]}|\varphi(t)-\psi(t)|
$$

for all $\varphi, \psi \in C[0,1]$. Then, $(C[0,1], \varrho)$ is a complete metric space.

We assume the following:

$\left(A_{1}\right)$ For each $\varphi \in C[0,1]$, the operator $K:[0,1] \times[0,1] \times \mathbb{R} \rightarrow K_{c v}(\mathbb{R})$ is such that $K(t, s, \varphi(s))$ is lower semicontinuous in $[0,1] \times[0,1]$.

$\left(A_{2}\right)$ There exists a continuous function $l:[0,1] \times[0,1] \rightarrow[0,+\infty)$ such that

$$
\left|k_{\varphi}(t, s)-k_{\psi}(t, s)\right| \leq l(t, s)\left\{\begin{array}{c}
\max \{|\varphi(s)-\psi(s)|,|\varphi(s)-K(t, s, \varphi(s))|, \\
|\psi(s)-K(t, s, \psi(s))|,|\varphi(s)-K(t, s, \psi(s))|,|\psi(s)-K(t, s, \varphi(s))|\}
\end{array}\right\}
$$

for all $t, s \in[0,1], \varphi, \psi \in C[0,1]$.

$\left(A_{3}\right)$ There exists some $k \in(0,1)$ such that

$$
\sup _{t \in[0,1]} \int_{0}^{1} l(t, s) \varrho s \leq k
$$

Theorem 4. Under Conditions $\left(A_{1}\right)-\left(A_{3}\right)$, the integral inclusion in (22) has a solution in $C[0,1]$.

Proof. Let $\mathcal{R}=C[0,1]$. Define the fuzzy mapping $\mathcal{Q}: \mathcal{R} \rightarrow(\mathcal{R})$ by

$$
[\mathcal{Q} \varphi]_{\alpha_{\mathcal{Q}}(\varphi)}=\left\{\psi \in \mathcal{R}: \psi(t) \in f(t)+\int_{0}^{1} K(t, s, \varphi(s)) \varrho s, t \in[0,1]\right\} .
$$

It is clear that the set of solutions of the integral inclusion in (22) coincides with the set of fixed points of the operator $\mathcal{Q}$ (see $[29,30]$ ). Hence, we have to prove that under the given conditions, $\mathcal{Q}$ has at least one fixed point in $\mathcal{R}$. For this, we check that the conditions of Corollary 11 hold true.

Let $\varphi \in \mathcal{R}$ be arbitrary; then, there exists $\alpha_{\mathcal{Q}}(\varphi) \in(0,1]$. For the multivalued operator $K(t, s)$ : $[0,1] \times[0,1] \rightarrow K_{c v}(\mathbb{R})$, it follows from the Michael's selection theorem that there exists a continuous operator $k_{\varphi}(t, s):[0,1] \times[0,1] \rightarrow \mathbb{R}$ such that $k_{\varphi}(t, s) \in K_{\varphi}(t, s)$ for each $t, s \in[0,1]$. It follows that $f(t)+\int_{0}^{1} k_{\varphi}(t, s) \varrho s \in[\mathcal{Q} \varphi]_{\mathcal{Q}_{\mathcal{Q}}(\varphi)}$. Hence, $[\mathcal{Q} \varphi]_{\alpha_{\mathcal{Q}}(\varphi)} \neq \varnothing$. It is an easy matter to show that $[\mathcal{Q} \varphi]_{\mathcal{Q}_{\mathcal{Q}}(\varphi)}$ is closed, and thus the details are omitted (see also [31,32]). Moreover, since $f$ is continuous on $[0,1]$ and $K_{\varphi}(t, s)$ is continuous on $[0,1] \times[0,1]$, their ranges are bounded. It follows that $[\mathcal{Q} \varphi]_{\mathcal{Q}_{\mathcal{Q}}(\varphi)}$ is also bounded. Hence, $[\mathcal{Q} \varphi]_{\alpha_{\mathcal{Q}}(\varphi)} \in C B(\mathcal{R})$. 
Let $\varphi_{1}, \varphi_{2} \in \mathcal{R}$; then, there exists $\alpha_{\mathcal{Q}}\left(\varphi_{1}\right), \alpha_{\mathcal{Q}}\left(\varphi_{1}\right) \in(0,1]$ such that $\left[\mathcal{Q} \varphi_{1}\right]_{\alpha_{\mathcal{Q}}\left(\varphi_{1}\right)},\left[\mathcal{Q}_{2}\right]_{\alpha_{\mathcal{Q}}\left(\varphi_{2}\right)}$ are nonempty, closed and bounded subsets of $\mathcal{R}$. Let $\psi_{1} \in\left[\mathcal{Q}_{1}\right]_{\alpha_{\mathcal{Q}}\left(\varphi_{1}\right)}$ be arbitrary such that

$$
\psi_{1}(t) \in f(t)+\int_{0}^{1} K\left(t, s, \varphi_{1}(s)\right) \varrho s
$$

for $t \in[0,1]$ holds. This means that for all $t, s \in[0,1]$, there exists $k_{\varphi_{1}}(t, s) \in K_{\varphi_{1}}(t, s)=K\left(t, s, \varphi_{1}(s)\right)$ such that

$$
\psi_{1}(t)=f(t)+\int_{0}^{1} k_{\varphi_{1}}(t, s) \varrho s
$$

for $t \in[0,1]$. For all $\varphi_{1}, \varphi_{2} \in \mathcal{R}$, it follows from $\left(A_{2}\right)$ that

$$
H\left(K\left(t, s, \varphi_{1}\right)-K\left(t, s, \varphi_{2}\right) \leq l(t, s)\left\{\begin{array}{c}
\max \left\{\left|\varphi_{1}(s)-\varphi_{2}(s)\right|,\left|\varphi_{1}(s)-K\left(t, s, \varphi_{1}(s)\right)\right|,\right. \\
\left|\varphi_{2}(s)-K\left(t, s, \varphi_{2}(s)\right)\right|,\left|\varphi_{1}(s)-K\left(t, s, \varphi_{2}(s)\right)\right|, \\
\left.\left|\varphi_{2}(s)-K\left(t, s, \varphi_{1}(s)\right)\right|\right\}
\end{array}\right\} .\right.
$$

This means that there exists $z(t, s) \in K_{\varphi_{2}}(t, s)$ such that

$$
\left|k_{\varphi_{1}}(t, s)-z(t, s)\right| \leq l(t, s)\left\{\begin{array}{c}
\max \left\{\left|\varphi_{1}(s)-\varphi_{2}(s)\right|,\left|\varphi_{1}(s)-K\left(t, s, \varphi_{1}(s)\right)\right|,\right. \\
\left|\varphi_{2}(s)-K\left(t, s, \varphi_{2}(s)\right)\right|,\left|\varphi_{1}(s)-K\left(t, s, \varphi_{2}(s)\right)\right|, \\
\left.\left|\varphi_{2}(s)-K\left(t, s, \varphi_{1}(s)\right)\right|\right\}
\end{array}\right\} .
$$

for all $t, s \in[0,1]$.

Now, we can consider the multivalued operator $U$ defined by

$$
U(t, s)=K_{\varphi_{2}}(t, s) \cap\left\{u \in \mathbb{R}:\left|k_{\varphi_{1}}(t, s)-u\right| \leq l(t, s)\left|\varphi_{1}(s)-\varphi_{2}(s)\right|\right\} .
$$

Hence, by $\left(A_{1}\right), U$ is lower semicontinuous; it follows that there exists a continuous operator $k_{\varphi_{2}}(t, s):[0,1] \times[0,1] \rightarrow \mathbb{R}$ such that $k_{\varphi_{2}}(t, s) \in U(t, s)$ for $t, s \in[0,1]$. Then, $\psi_{2}(t)=f(t)+\int_{0}^{1} k_{\varphi_{1}}(t, s) \varrho s$ satisfies that

$$
\psi_{2}(t) \in f(t)+\int_{0}^{1} K\left(t, s, \varphi_{2}(s)\right) \varrho s, \quad t \in[0,1]
$$

$t \in[0,1]$. That is, $\psi_{2} \in\left[\mathcal{Q} \varphi_{2}\right]_{\alpha_{\mathcal{Q}}\left(\varphi_{2}\right)}$ and

$$
\begin{aligned}
\left|\psi_{1}(t)-\psi_{2}(t)\right| & \leq \int_{0}^{1}\left|k_{\varphi_{1}}(t, s)-k_{\varphi_{2}}(t, s)\right| \varrho s \\
& \leq \int_{0}^{1} l(t, s)\left|\varphi_{1}(s)-\varphi_{2}(s)\right| \varrho s \\
& \leq \max _{t \in[0,1]}\left(\int_{0}^{1} l(t, s)\left\{\begin{array}{c}
\max \left\{\left|\varphi_{1}(s)-\varphi_{2}(s)\right|,\left|\varphi_{1}(s)-K\left(t, s, \varphi_{1}(s)\right)\right|,\right. \\
\left|\varphi_{2}(s)-K\left(t, s, \varphi_{2}(s)\right)\right|,\left|\varphi_{1}(s)-K\left(t, s, \varphi_{2}(s)\right)\right|, \\
\left.\left|\varphi_{2}(s)-K\left(t, s, \varphi_{1}(s)\right)\right|\right\}
\end{array}\right\} \varrho s\right. \\
& \leq k \max \left\{\begin{array}{c}
\varrho\left(\varphi_{1}, \varphi_{2}\right), \varrho\left(\varphi_{1},\left[\mathcal{Q} \varphi_{1}\right]_{\alpha_{\mathcal{Q}}\left(\varphi_{1}\right)}\right), \varrho\left(\varphi_{2},\left[\mathcal{Q} \varphi_{2}\right]_{\alpha_{\mathcal{Q}}\left(\varphi_{2}\right)}\right), \\
\varrho\left(\varphi_{1},\left[\mathcal{Q} \varphi_{2}\right]_{\mathcal{Q}_{\mathcal{Q}}\left(\varphi_{2}\right)}\right), \varrho\left(\varphi_{2},\left[\mathcal{Q} \varphi_{1}\right]_{\alpha_{\mathcal{Q}}\left(\varphi_{1}\right)}\right)
\end{array}\right\}
\end{aligned}
$$

for all $t, s \in[0,1]$. Thus, we obtain that

$$
\varrho\left(\psi_{1}, \psi_{2}\right) \leq k \max \left\{\begin{array}{c}
\varrho\left(\varphi_{1}, \varphi_{2}\right), \varrho\left(\varphi_{1},\left[\mathcal{Q} \varphi_{1}\right]_{\alpha_{\mathcal{Q}}\left(\varphi_{1}\right)}\right), \varrho\left(\varphi_{2},\left[\mathcal{Q} \varphi_{2}\right]_{\alpha_{\mathcal{Q}}\left(\varphi_{2}\right)}\right) \\
\varrho\left(\varphi_{1},\left[\mathcal{Q} \varphi_{2}\right]_{\alpha_{\mathcal{Q}}\left(\varphi_{2}\right)}\right), \varrho\left(\varphi_{2},\left[\mathcal{Q} \varphi_{1}\right]_{\alpha_{\mathcal{Q}}\left(\varphi_{1}\right)}\right)
\end{array}\right\} .
$$


Interchanging the roles of $\varphi_{1}$ and $\varphi_{2}$, we obtain that

$$
H\left(\left[\mathcal{Q} \varphi_{1}\right]_{\alpha_{\mathcal{Q}}\left(\varphi_{1}\right)},\left[\mathcal{Q} \varphi_{2}\right]_{\alpha_{\mathcal{Q}}\left(\varphi_{2}\right)}\right) \leq k \max \left\{\begin{array}{c}
\varrho\left(\varphi_{1}, \varphi_{2}\right), \varrho\left(\varphi_{1},\left[\mathcal{Q} \varphi_{1}\right]_{\alpha_{\mathcal{Q}}\left(\varphi_{1}\right)}\right), \varrho\left(\varphi_{2},\left[\mathcal{Q} \varphi_{2}\right]_{\alpha_{\mathcal{Q}}\left(\varphi_{2}\right)}\right) \\
\varrho\left(\varphi_{1},\left[\mathcal{Q} \varphi_{2}\right]_{\alpha_{\mathcal{Q}}\left(\varphi_{2}\right)}\right), \varrho\left(\varphi_{2},\left[\mathcal{Q} \varphi_{1}\right]_{\alpha_{\mathcal{Q}}\left(\varphi_{1}\right)}\right)
\end{array}\right\}
$$

Taking exponential on both side, we have

$$
e^{H\left(\left[\mathcal{Q} \varphi_{1}\right]_{\alpha_{\mathcal{Q}}}\left(\varphi_{1}\right),\left[\mathcal{Q} \varphi_{2}\right]_{\alpha_{\mathcal{Q}}}\left(\varphi_{2}\right)\right.} \leq e^{k \max \left\{\varrho\left(\varphi_{1}, \varphi_{2}\right), \varrho\left(\varphi_{1},\left[\mathcal{Q} \varphi_{1}\right]_{\mathcal{Q}_{\mathcal{Q}}}\left(\varphi_{1}\right)\right), Q\left(\varphi_{2},\left[\mathcal{Q} \varphi_{2}\right]_{\alpha_{\mathcal{Q}}}\left(\varphi_{2}\right), \rho\left(\varphi_{1},\left[\mathcal{Q} \varphi_{2}\right]_{\alpha_{\mathcal{Q}}}\left(\varphi_{2}\right), \varrho\left(\varphi_{2},\left[\mathcal{Q} \varphi_{1}\right]_{\alpha_{\mathcal{Q}}}\left(\varphi_{1}\right)\right\}\right.\right.\right.} .
$$

Taking the function $\rho \in \mathcal{P}$ given by $\rho\left(\varphi_{1}, \varphi_{2}, \varphi_{3}, \varphi_{4}, \varphi_{5}\right)=\max \left\{\varphi_{1}, \varphi_{2}, \varphi_{3}, \varphi_{4}, \varphi_{5}\right\}$ and $\Theta \in \digamma$ defined by $\Theta(t)=e^{\sqrt{t}}$ for $t>0$, we obtain that the condition in (21) is fulfilled. Using Corollary 11, we conclude that the given integral inclusion has a solution.

\section{Conclusions}

In this paper, we obtain certain common fixed point theorems for $\alpha$-fuzzy mappings satisfying a new class of contractive conditions in the setting of complete metric spaces. We derive various well known results of the literature as corollaries. The existence of solutions for the Fredholm integral inclusion is also investigated as application of our main result. We also establish a significant example to support our results. We hope that the results contained in this paper will raise new associations for those who are working in $\Theta$-contraction, fuzzy mapping and its applications to integral inclusions.

Author Contributions: All authors contributed equally and significantly in writing this paper. All authors read and approved the final paper.

Acknowledgments: This project was funded by the Deanship of Scientific Research (DSR), King Abdulaziz University, Jeddah, Saudi Arabia under grant no. (KEP-23-130-38). The authors, therefore, acknowledge with thanks DSR technical and financial support.

Conflicts of Interest: The authors declare that they have no competing interests.

\section{References}

1. Banach, S. Sur les opérations dans les ensembles abstraits et leur application aux équations intégrales. Fund. Math. 1922, 3, 133-181. [CrossRef]

2. Jleli, M.; Samet, B. A new generalization of the Banach contraction principle. J. Inequal. Appl. $2014,2014,38$. [CrossRef]

3. Ahmad, J.; Al-Mazrooei, A.E.; Altun, I. Generalized $\Theta$-contractive fuzzy mappings. J. Intell. Fuzzy Syst. 2018, 35, 1935-1942. [CrossRef]

4. Al-Rawashdeh. A.; Ahmad, J. Common Fixed Point Theorems for JS- Contractions. Bull. Math. Anal. Appl. 2016, $8,12-22$.

5. Hussain, N.; Ahmad, J.; Ćirić. L.; Azam, A. Coincidence point theorems for generalized contractions with application to integral equations. Fixed Point Theory Appl. 2015, 2015, 78. [CrossRef]

6. Vetro, F. A generalization of Nadler fixed point theorem. Carpathian J. Math. 2015, 31, 403-410.

7. Al-Mazrooei, A.E.; Ahmad, J. Fixed Point Theorems for Fuzzy Mappings with Applications. J. Intell. Fuzzy Syst. 2019, 36, 3903-3909. [CrossRef]

8. Hussain, N.; Parvaneh, V.; Samet. B.; Vetro, C. Some fixed point theorems for generalized contractive mappings in complete metric spaces. Fixed Point Theory Appl. 2015, 2015, 185. [CrossRef]

9. Nadler, S.B., Jr. Multivalued contraction mappings. Pac. J. Math. 1969, 30, 475-478. [CrossRef]

10. Constantin, A. A random fixed point theorem for multifunctions. Stoch. Anal. Appl. 1994, 12, 65-73. [CrossRef]

11. Isik, H. Fractional Differential Inclusions with a New Class of Set-Valued Contractions. arXiv 2018, arXiv:1807.05427. 
12. Heilpern, S. Fuzzy mappings and fixed point theorem. J. Math. Anal. Appl. 1981, 83, 566-569. [CrossRef]

13. Adibi, H.; Cho, Y.J.; O'Regan, D.; Saadati, R. Common fixed point theorems in L-fuzzy metric spaces. Appl. Math. Comput. 2006, 182, 820-828. [CrossRef]

14. Arora, S.C.; Sharma, V. Fixed points for fuzzy mappings. Fuzzy Sets Syst. 2000, 110, 127-130. [CrossRef]

15. Azam, A.; Beg, I. Common fixed points of fuzzy maps. Math. Comput. Model. 2009, 49, 1331-1336. [CrossRef]

16. Azam, A.; Arshad, M.; Vetro, P. On a pair of fuzzy $\varphi$-contractive mappings. Math. Comput. Model. 2010, 52, 207-214. [CrossRef]

17. Azam, A. Fuzzy Fixed Points of Fuzzy Mappings via a Rational Inequality. Hacet. J. Math. Stat. 2011, 40, 421-431.

18. Ahmad, J.; Azam, A.; Romaguera, S. On locally contractive fuzzy set-valued mappings. J. Inequal. Appl. 2014, 2014, 74. [CrossRef]

19. Bose, R.K.; Sahani, D. Fuzzy mappings and fixed point theorems. Fuzzy Sets Syst. 1987, 21, 53-58. [CrossRef]

20. Chang, S.S.; Cho, Y.J.; Lee, B.S.; Jung, J.S. Kang, S.M. Coincidence point and minimization theorems in fuzzy metric spaces. Fuzzy Sets Syst. 1997, 88, 119-128. [CrossRef]

21. Kannan, R. Some results on fixed points. Bull. Calcutta Math. Soc. 1968, 60, 71-76.

22. Chatterjea, S.K. Fixed point theorems. C. R. Acad. Bulgare Sci. 1972, 25, 727-730. [CrossRef]

23. Reich, S. Kannan's fixed point theorem. Boll. Un. Mat. Ital. 1971, 4, 1-11.

24. Reich, S. Fixed points of contractive functions. Boll. dell'Unione Mat. Italy. 1972, 4, $26-42$.

25. Berinde, V. Approximating fixed points of weak contractions using the Picard iteration. Nonlinear Anal. Forum 2004, 9, 43-53.

26. Hardy, G.E.; Rogers, T.D. A generalization of a fixed point theorem of Reich. Can. Math. Bull. 1973, 16, $201-206$. [CrossRef]

27. Ćirić, L.B. A generalization of Banach's contraction principle. Proc. Am. Math. Soc. 1974, 45, $267-273$.

28. Ćirić, L.B. Generalized contractions and fixed point theorems. Pub. Inst. Math. (Belgrad) 1971, 12, $19-26$.

29. Agarwal, R.P.; Hussain, N.; Taoudi, M.-A. Fixed point theorems in ordered Banach spaces and applications to nonlinear integral equations. Abstr. Appl. Anal. 2012, 2012, 1-15. [CrossRef]

30. Nieto, J.J.; Rodríguez-López, R. Existence and uniqueness of fixed point in partially ordered sets and applications to ordinary differential equations. Acta Math. Sin. 2017, 23, 2205-2212. [CrossRef]

31. Shi-sheng, Z. Fixed point theorems for fuzzy mappings (II). Appl. Math. Mech. 1986, 7, 147-152. [CrossRef]

32. Sîntamarian, A. Integral inclusions of Fredholm type relative to multivalued $\varphi$-contractions. Semin. Fixed Point Theory Cluj Napoca 2002, 3, 361-368.

(C) 2019 by the authors. Licensee MDPI, Basel, Switzerland. This article is an open access article distributed under the terms and conditions of the Creative Commons Attribution (CC BY) license (http:/ / creativecommons.org/licenses/by/4.0/). 